\title{
Stability limit of human-in-the-loop model reference adaptive control architectures
}

\author{
Tansel Yucelen $\oplus^{a}$, Yildiray Yildiz ${ }^{b}$, Rifat Sipahic ${ }^{c}$, Ehsan Yousefib and Nhan Nguyen ${ }^{d}$ \\ ${ }^{a}$ Department of Mechanical Engineering, University of South Florida, Tampa, FL, USA; ${ }^{b}$ Department of Mechanical Engineering, Bilkent \\ University, Ankara, Turkey; ${ }^{C}$ Department of Mechanical and Industrial Engineering, Northeastern University, Boston, MA, USA; ${ }^{d}$ Intelligent \\ Systems Division, National Aeronautics and Space Administration, Moffett Field, CA, USA
}

\begin{abstract}
Model reference adaptive control (MRAC) offers mathematical and design tools to effectively cope with many challenges of real-world control problems such as exogenous disturbances, system uncertainties and degraded modes of operations. On the other hand, when faced with human-in-the-loop settings, these controllers can lead to unstable system trajectories in certain applications. To establish an understanding of stability limitations of MRAC architectures in the presence of humans, here a mathematical framework is developed whereby an MRAC is designed in conjunction with a class of linear human models including human reaction delays. This framework is then used to reveal, through stability analysis tools, the stability limit of the MRAC-human closed-loop system and the range of model parameters respecting this limit. An illustrative numerical example of an adaptive flight control application with a Neal-Smith pilot model is presented to demonstrate the effectiveness of developed approaches.
\end{abstract}

\section{ARTICLE HISTORY}

Received 23 August 2016

Accepted 10 June 2017

\section{KEYWORDS}

Uncertain dynamical systems; model reference adaptive control; human-in-the-loop systems; closed-loop system stability; human reaction time delay

\section{Introduction}

Achieving system stability and a level of desired system performance when dealing with uncertain dynamical systems is one of the major challenges arising in control theory. While fixed-gain robust control design approaches (Skogestad \& Postlethwaite, 2007; Weinmann, 2012; Zhou \& Doyle, 1998; Zhou et al., 1996) can deal with such dynamical systems, the knowledge of system uncertainty bounds is required and characterisation of these bounds is not trivial in general due to practical constraints such as extensive and costly verification and validation procedures. On the other hand, adaptive control design approaches (Åström \& Wittenmark, 2013; Ioannou \& Sun, 2012; Lavretsky \& Wise, 2012; Narendra \& Annaswamy, 2012) are important candidates for uncertain dynamical systems since they can effectively cope with the effects of system uncertainties online and require less modelling information than fixed-gain robust control design approaches (Yucelen, De La Torre, \& Johnson, 2014; Yucelen \& Haddad, 2012).

This paper builds on one of the well-known and important class of adaptive controllers; namely model reference adaptive controllers (MRACs) (Osburn, Whitaker, \& Kezer, 1961; Whitaker, Yamron, \& Kezer, 1958), where their architecture includes a reference model, a parameter adjustment mechanism and a controller. In this setting, a desired closed-loop dynamical system behaviour is captured by the reference model, where its output (respectively, state) is compared with the output (respectively, state) of the uncertain dynamical system. This comparison yields a system error signal, which is used to drive an online parameter adjustment mechanism. Then, the controller adapts feedback gains to minimise this error signal using the information received from the parameter adjustment mechanism. As a consequence, under proper settings, the output (respectively, state) of the uncertain dynamical system behaves as the output (respectively, state) of the reference model asymptotically or approximately in time, and hence, guarantees system stability and achieves a level of desired closed-loop dynamical system behaviour.

While MRAC offers mathematical and design tools to effectively cope with system uncertainties arising from ideal assumptions (e.g. linearisation, model order reduction, exogenous disturbances and degraded modes of operations), its capabilities when interfaced with human operators can, however, be quite limited. Indeed, in certain applications when humans are in the loop (Miller, 2011; Ryu \& Andrisani, 2003; Trujillo \& Gregory, 2013; Trujillo, Gregory, \& Hempley, 2015), the arising closed loop with MRAC can become unstable. As a matter of fact, such problems are not only limited to 
MRAC-human interactions and have been reported to arise in various human-in-the-loop control problems including, for example, pilot-induced oscillations (Klyde \& McRuer, 2009). To address these issues, novel control design ideas were proposed and/or experimentally tested including adaptive control as well as smart-cue/smartgain concepts (Klyde \& McRuer, 2009; Richards, Adams, Klyde, \& Cogan, 2015). On the other hand, an analytical framework aimed at understanding these phenomena and that can ultimately be used to drive rigorous control design is currently lacking. These observations motivate this study where the main objective is to develop comprehensive models from a system-level perspective and analyse such models to develop a strong understanding of the aforementioned stability limits, in particular within the framework of human-in-the-loop MRAC architectures.

With the human-in-the-loop, one critical parameter added to the control problem that can be responsible for instabilities is the human reaction delays - a topic which has been long investigated (Green, 2000; Helbing, 2001; McRuer, 1974; Stépán, 2009; Treiber, Kesting, \& Helbing, 2006) including adaptive control of time-delay systems (Bekiaris-Liberis \& Krstic, 2010; Bresch-Pietri \& Krstic, 2009; Krstic, 1994; Niculescu \& Annaswamy, 2003; Ortega \& Lozano, 1988; Yildiz, Annaswamy, Kolmanovsky, \& Yanakiev, 2010), but not thoroughly in the context of human-in-the-loop adaptive control. It is known that the presence of time delays is a source of instability, which must be carefully dealt with and explicitly addressed in any control design framework (Bellman \& Cooke, 1963; Stépán, 1989). Delay-induced instability phenomenon has been recognised in numerous applications including robotics, physics, cyber-physical systems and operational psychology (Sipahi, Niculescu, Abdallah, Michiels, \& Gu, 2011). For example, in physics literature, effects of human decision-making process and reaction delays are studied to explain the arising car driving patterns, traffic flow characteristics, traffic jams, and stopand-go waves (Bando, Hasebe, Nakanishi, \& Nakayama, 1998; Helbing, 2001).

In terms of mathematical modelling of human behaviour, many studies focus on developing a representative transfer function of the human in a specific task within a certain frequency band. Along these lines, we cite three key models; (i) human driver models (Helbing, 2001), (ii) McRuer cross-over model (McRuer, 1974), and (iii) Neal-Smith pilot model; for example, see Schmidt and Bacon (1983), Thurling (2000), Ryu and Andrisani (2003) and Witte (2004), Miller (2011). Human driver models are proposed in the context of car driving, specifically in longitudinal car-following tasks in a fixed lane. While these models vary depending on the degree of their complexity, for example, see Treiber et al. (2006), their simplest form is a pure time delay representing the dead time between arrival of stimulus and reaction produced by the driver. McRuer's model was, on the other hand, proposed to capture human pilot behaviour, to further understand flight stability and human-vehicle integration. Among many of its variations, this model is essentially an integrator dynamics with a time lag to capture human reaction delays and a gain modulated to maintain a specific bandwidth. Similarly, the Neal-Smith pilot model, which is essentially a first-order lead-lag-type compensator with a gain and time lag, can be utilised to study the behaviour of human pilots (see the above-cited references).

In light of the above discussions, it is of strong interest to understand the limitations of MRAC when coupled with human operators in a closed-loop setting. For this purpose, here MRAC is first incorporated into a general linear human model with reaction delays. Through the use of stability theory, this model is then studied to reveal its fundamental stability limit, and the parameter space of the model where such limit is respected hence MRAChuman-combined model produces stable trajectories. An illustrative numerical example of an adaptive flight control application with a Neal-Smith pilot model and uncertainties is presented next to demonstrate the effectiveness of developed approaches.

The main contribution of this study is the development of a comprehensive control-theoretic modelling approach, where the dynamic interactions between a general class of human models and MRAC framework can be investigated. We particularly focus on understanding how an ideal MRAC would perform in conjunction with a human model including human reaction delays and how such delays could pose strong limitations to the stabilisation and performance of the arising closedloop human-MRAC architecture. To this end, we lay out the approaches and the pertaining theory with rigorous proofs guaranteeing stability independent of delays and conditions under which stability can be lost. These results pave the way towards studying more complex human models with MRAC, advancing the design of MRAC to better accommodate human dynamics, and driving experimental studies with an analytical foundation.

The notation used in this paper is standard. Specifically, $\mathbb{R}$ denotes the set of real numbers, $\mathbb{R}^{n}$ denotes the set of $n \times 1$ real column vectors, $\mathbb{R}^{n \times m}$ denotes the set of $n \times m$ real matrices, $\mathbb{R}_{+}$(resp., $\overline{\mathbb{R}}_{+}$) denotes the set of positive (resp., non-negative-definite) real numbers, $\mathbb{R}_{+}^{n \times n}$ (resp., $\overline{\mathbb{R}}_{+}^{n \times n}$ ) denotes the set of $n \times$ $n$ positive-definite (resp., non-negative-definite) real matrices, $\mathbb{S}^{n \times n}$ denotes the set of $n \times n$ symmetric real matrices, $\mathbb{D}^{n \times n}$ denotes the $n \times n$ real matrices with 


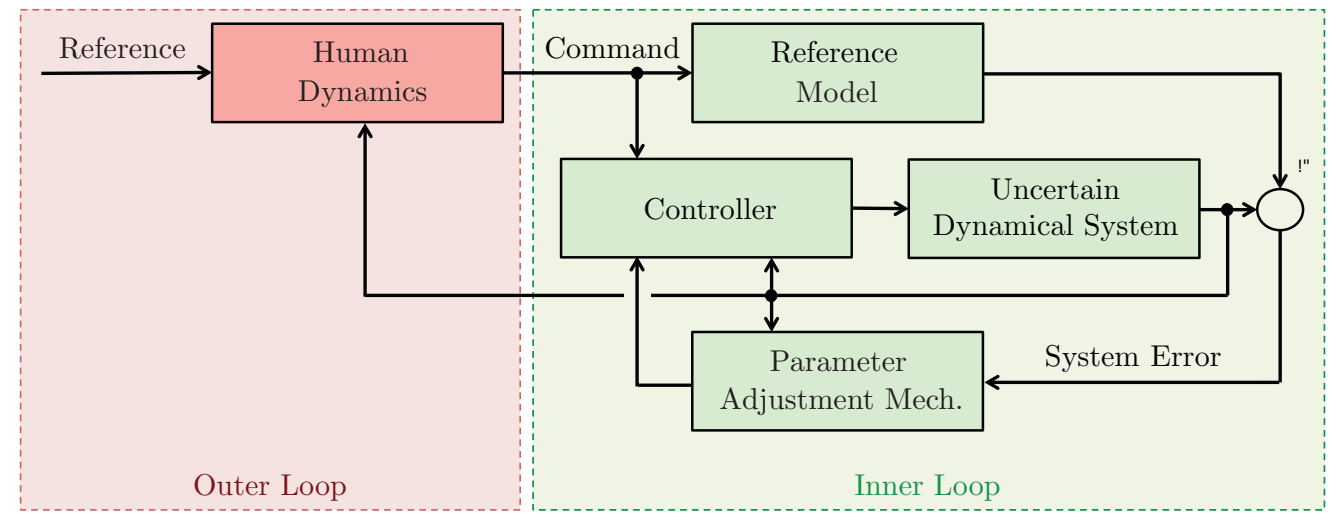

Figure 1. Block diagram of the human-in-the-loop model reference adaptive control architecture.

diagonal scalar entries, $(\cdot)^{\mathrm{T}}$ denotes transpose, $(\cdot)^{-1}$ denotes inverse, and ' $\triangleq$ ' denotes equality by definition. In addition, we write $\lambda_{\min }(A)$ (resp., $\left.\lambda_{\max }(A)\right)$ for the minimum (resp., maximum) eigenvalue of the matrix $A$, $\operatorname{tr}(\cdot)$ for the trace operator, $\operatorname{vec}(\cdot)$ for the column stacking operator, $\|\cdot\|_{2}$ for the Euclidian norm, $\|\cdot\|_{\infty}$ for the infinity norm, and $\|\cdot\|_{F}$ for the Frobenius matrix norm.

\section{Problem formulation}

To study human-in-the-loop model reference adaptive controllers, we start with the block diagram configuration given by Figure 1. In the figure, the outer-loop architecture includes the reference that is fed into the human dynamics to generate a command for the innerloop architecture in response to the variations resulting from the uncertain dynamical system. In this setting, the reference input is what the human aims to achieve in a task, and the uncertain dynamical system is the machine on which this task is being performed. The inner-loop architecture includes the uncertain dynamical system as well as the model reference adaptive controller components (i.e. the reference model, the parameter adjustment mechanism and the controller). Specifically, at the outerloop architecture, we consider a general class of linear human models with constant time delay given by

$$
\begin{aligned}
\dot{\xi}(t) & =A_{\mathrm{h}} \xi(t)+B_{\mathrm{h}} \theta(t-\tau), \quad \xi(0)=\xi_{0}, \\
c(t) & =C_{\mathrm{h}} \xi(t)+D_{\mathrm{h}} \theta(t-\tau),
\end{aligned}
$$

where $\xi(t) \in \mathbb{R}^{n_{\xi}}$ is the internal human state vector, $\tau \in$ $\mathbb{R}_{+}$is the internal human time delay, $A_{\mathrm{h}} \in \mathbb{R}^{n_{\xi} \times n_{\xi}}, B_{\mathrm{h}} \in$ $\mathbb{R}^{n_{\xi} \times n_{\mathrm{r}}}, C_{\mathrm{h}} \in \mathbb{R}^{n_{\mathrm{c}} \times n_{\xi}}, D_{\mathrm{h}} \in \mathbb{R}^{n_{\mathrm{c}} \times n_{\mathrm{r}}}$ and $c(t) \in \mathbb{R}^{n_{\mathrm{c}}}$ is the command produced by the human, which is the input to the inner-loop architecture as shown in Figure 1. Here, input to the human dynamics is given by

$$
\theta(t) \triangleq r(t)-E_{\mathrm{h}} x(t)
$$

where $\theta(t) \in \mathbb{R}^{n_{\mathrm{r}}}$, with $r(t) \in \mathbb{R}^{n_{\mathrm{r}}}$ being the bounded reference. Here $x(t) \in \mathbb{R}^{n}$ is the state vector (further details below) and $E_{\mathrm{h}} \in \mathbb{R}^{n_{\mathrm{r}} \times n}$ selects the appropriate states to be compared with $r(t)$. Note that the dynamics given by (1)(3) is general enough to capture, for example, widely studied linear time-invariant human models with time-delay including Neal-Smith model and its extensions (Miller, 2011; Ryu \& Andrisani, 2003; Schmidt \& Bacon, 1983; Thurling, 2000; Witte, 2004).

Next, at the inner-loop architecture, we consider the uncertain dynamical system given by

$$
\begin{gathered}
\dot{x}_{\mathrm{p}}(t)=A_{\mathrm{p}} x_{\mathrm{p}}(t)+B_{\mathrm{p}} \Lambda u(t)+B_{\mathrm{p}} \delta_{\mathrm{p}}\left(x_{\mathrm{p}}(t)\right), \\
x_{\mathrm{p}}(0)=x_{\mathrm{p}_{0}},
\end{gathered}
$$

where $x_{\mathrm{p}}(t) \in \mathbb{R}^{n_{\mathrm{p}}}$ is the accessible state vector, $u(t) \in$ $\mathbb{R}^{m}$ is the control input, $\delta_{\mathrm{p}}: \mathbb{R}^{n_{\mathrm{p}}} \rightarrow \mathbb{R}^{m}$ is an uncertainty, $A_{\mathrm{p}} \in \mathbb{R}^{n_{\mathrm{p}} \times n_{\mathrm{p}}}$ is a known system matrix, $B_{\mathrm{p}} \in \mathbb{R}^{n_{\mathrm{p}} \times m}$ is a known control input matrix and $\Lambda \in \mathbb{R}_{+}^{m \times m} \cap \mathbb{D}^{m \times m}$ is an unknown control effectiveness matrix. Furthermore, we assume that the pair $\left(A_{\mathrm{p}}, B_{\mathrm{p}}\right)$ is controllable and the uncertainty is parameterised as

$$
\delta_{\mathrm{p}}\left(x_{\mathrm{p}}\right)=W_{\mathrm{p}}^{\mathrm{T}} \sigma_{\mathrm{p}}\left(x_{\mathrm{p}}\right), \quad x_{\mathrm{p}} \in \mathbb{R}^{n_{\mathrm{p}}},
$$

where $W_{\mathrm{p}} \in \mathbb{R}^{s \times m}$ is an unknown weight matrix and $\sigma_{\mathrm{p}}: \mathbb{R}^{n_{\mathrm{p}}} \rightarrow \mathbb{R}^{s}$ is a known basis function of the form $\sigma_{\mathrm{p}}\left(x_{\mathrm{p}}\right)=\left[\sigma_{\mathrm{p}_{1}}\left(x_{\mathrm{p}}\right), \sigma_{\mathrm{p}_{2}}\left(x_{\mathrm{p}}\right), \ldots, \sigma_{\mathrm{p}_{\mathrm{s}}}\left(x_{\mathrm{p}}\right)\right]^{\mathrm{T}}$.

Remark 2.1: Note for the case where the basis function $\sigma_{\mathrm{p}}\left(x_{\mathrm{p}}\right)$ is unknown, the parameterisation in (5) can be relaxed (Lewis, Liu, \& Yesildirek, 1995; Lewis, Yesildirek, \& Liu, 1996) without significantly changing the results of 
this paper by considering

$$
\delta_{\mathrm{p}}\left(x_{\mathrm{p}}\right)=W_{\mathrm{p}}^{\mathrm{T}} \sigma_{\mathrm{p}}^{\mathrm{nn}}\left(V_{\mathrm{p}}^{\mathrm{T}} x_{\mathrm{p}}\right)+\varepsilon_{\mathrm{p}}^{\mathrm{nn}}\left(x_{\mathrm{p}}\right), \quad x_{\mathrm{p}} \in \mathcal{D}_{x_{\mathrm{p}}},
$$

where $W_{\mathrm{p}} \in \mathbb{R}^{s \times m}$ and $V_{\mathrm{p}} \in \mathbb{R}^{n_{\mathrm{p}} \times s}$ are unknown weight matrices, $\sigma_{\mathrm{p}}^{\mathrm{nn}}: \mathcal{D}_{x_{\mathrm{p}}} \rightarrow \mathbb{R}^{s}$ is a known basis composed of neural networks function approximators, $\varepsilon_{\mathrm{p}}^{\mathrm{nn}}: \mathcal{D}_{x_{\mathrm{p}}} \rightarrow$ $\mathbb{R}^{m}$ is an unknown residual error, and $\mathcal{D}_{x_{\mathrm{p}}}$ is a compact subset of $\mathbb{R}^{n_{\mathrm{p}}}$.

To address command following at the inner-loop architecture, let $x_{\mathrm{c}}(t) \in \mathbb{R}^{n_{\mathrm{c}}}$ be the integrator state satisfying

$$
\dot{x}_{\mathrm{c}}(t)=E_{\mathrm{p}} x_{\mathrm{p}}(t)-c(t), \quad x_{\mathrm{c}}(0)=x_{\mathfrak{c}_{0}},
$$

where $E_{\mathrm{p}} \in \mathbb{R}^{n_{\mathrm{c}} \times n_{\mathrm{p}}}$ allows to choose a subset of $x_{\mathrm{p}}(t)$ to be followed by $c(t)$. Now, (4) can be augmented with (7) as

$$
\begin{aligned}
\dot{x}(t)= & A x(t)+B \Lambda u(t)+B W_{\mathrm{p}}^{\mathrm{T}} \sigma_{\mathrm{p}}\left(x_{\mathrm{p}}(t)\right) \\
& +B_{\mathrm{r}} c(t), \quad x(0)=x_{0},
\end{aligned}
$$

where

$$
\begin{aligned}
& A \triangleq\left[\begin{array}{cc}
A_{\mathrm{p}} & 0_{n_{\mathrm{p}} \times n_{\mathrm{c}}} \\
E_{\mathrm{p}} & 0_{n_{\mathrm{c}} \times n_{\mathrm{c}}}
\end{array}\right] \in \mathbb{R}^{n \times n}, \\
& B \triangleq\left[B_{\mathrm{p}}^{\mathrm{T}}, 0_{n_{\mathrm{c}} \times m}^{\mathrm{T}}\right]^{\mathrm{T}} \in \mathbb{R}^{n \times m}, \\
& B_{\mathrm{r}} \triangleq\left[0_{n_{\mathrm{p}} \times n_{\mathrm{c}}}^{\mathrm{T}},-I_{n_{\mathrm{c}} \times n_{\mathrm{c}}}\right]^{\mathrm{T}} \in \mathbb{R}^{n \times n_{\mathrm{c}}},
\end{aligned}
$$

and $x(t) \triangleq\left[x_{\mathrm{p}}^{\mathrm{T}}(t), x_{\mathrm{c}}^{\mathrm{T}}(t)\right]^{\mathrm{T}} \in \mathbb{R}^{n}$ is the augmented state vector, $x_{0} \triangleq\left[x_{\mathrm{p}_{0}}^{\mathrm{T}}, x_{\mathrm{c}_{0}}^{\mathrm{T}}\right]^{\mathrm{T}} \in \mathbb{R}^{n}$, and $n=n_{\mathrm{p}}+n_{\mathrm{c}}$. In this inner-loop architecture setting, without loss of theoretical generality, it is practically reasonable to set $E_{\mathrm{h}}=$ $\left[E_{\mathrm{h}_{\mathrm{p}}}, 0_{n_{\mathrm{r}} \times n_{\mathrm{c}}}\right]$ in (3) with $E_{\mathrm{h}_{\mathrm{p}}} \in \mathbb{R}^{n_{\mathrm{r}} \times n_{\mathrm{p}}}$, since a subset of the accessible state vector is usually available and/or sensed by the human at the outer loop (but not the states of the integrator).

Finally, consider the feedback control law at the innerloop architecture given by

$$
u(t)=u_{\mathrm{n}}(t)+u_{\mathrm{a}}(t),
$$

where $u_{\mathrm{n}}(t) \in \mathbb{R}^{m}$ and $u_{\mathrm{a}}(t) \in \mathbb{R}^{m}$ are the nominal and adaptive control laws, respectively. Furthermore, let the nominal control law be

$$
u_{\mathrm{n}}(t)=-K x(t)
$$

with $K \in \mathbb{R}^{m \times n}$, such that $A_{\mathrm{r}} \triangleq A-B K$ is Hurwitz. For instance, such $K$ exists if and only if $(A, B)$ is a controllable pair. Using (12) and (13) in (8) next yields

$$
\dot{x}(t)=A_{\mathrm{r}} x(t)+B_{\mathrm{r}} c(t)+B \Lambda\left[u_{\mathrm{a}}(t)+W^{\mathrm{T}} \sigma(x(t))\right],
$$

where $W^{\mathrm{T}} \triangleq\left[\Lambda^{-1} W_{\mathrm{p}}^{\mathrm{T}},\left(\Lambda^{-1}-I_{m \times m}\right) K\right] \in \mathbb{R}^{(s+n) \times m}$ is an unknown aggregated weight matrix and $\sigma^{\mathrm{T}}(x(t)) \triangleq$ $\left[\sigma_{\mathrm{p}}^{\mathrm{T}}\left(x_{\mathrm{p}}(t)\right), x^{\mathrm{T}}(t)\right] \in \mathbb{R}^{s+n}$ is a known aggregated basis function. Considering (14), let the adaptive control law be

$$
u_{\mathrm{a}}(t)=-\hat{W}^{\mathrm{T}}(t) \sigma(x(t))
$$

where $\hat{W}(t) \in \mathbb{R}^{(s+n) \times m}$ is the estimate of $W$ satisfying the parameter adjustment mechanism

$$
\dot{\hat{W}}(t)=\gamma \sigma(x(t)) e^{\mathrm{T}}(t) P B, \quad \hat{W}(0)=\hat{W}_{0},
$$

where $\gamma \in \mathbb{R}_{+}$is the learning rate, ${ }^{1}$ and system error reads

$$
e(t) \triangleq x(t)-x_{\mathrm{r}}(t)
$$

with $x_{\mathrm{r}}(t) \in \mathbb{R}^{n}$ being the reference state vector satisfying the reference system

$$
\dot{x}_{\mathrm{r}}(t)=A_{\mathrm{r}} x_{\mathrm{r}}(t)+B_{\mathrm{r}} c(t), \quad x_{\mathrm{r}}(0)=x_{\mathrm{r}_{0}},
$$

and $P \in \mathbb{R}_{+}^{n \times n} \cap \mathbb{S}^{n \times n}$ is a solution of the Lyapunov equation

$$
0=A_{\mathrm{r}}^{\mathrm{T}} P+P A_{\mathrm{r}}+R,
$$

with $R \in \mathbb{R}_{+}^{n \times n} \cap \mathbb{S}^{n \times n}$. Since $A_{\mathrm{r}}$ is Hurwitz, it follows from Haddad, Chellaboina, and Kablar (1999) that there exists a unique $P \in \mathbb{R}_{+}^{n \times n} \cap \mathbb{S}^{n \times n}$ satisfying (19) for a given $R \in \mathbb{R}_{+}^{n \times n} \cap \mathbb{S}^{n \times n}$.

Based on the given problem formulation, the next section analyses the stability of the coupled inner and outer-loop architectures depicted in Figure 1 in order to establish a fundamental stability limit for guaranteeing the closed-loop system stability. Specifically, it is of interest to reveal the conditions under which this limit is satisfied in terms of human model parameters at the outer loop and the given adaptive controller gains at the inner loop.

\section{Fundamental stability limit}

To analyse the stability of the coupled inner and outerloop architectures introduced in the previous section, we first write the system error dynamics using (14), (15) and 
(18) as

$$
\dot{e}(t)=A_{\mathrm{r}} e(t)-B \Lambda \tilde{W}^{\mathrm{T}}(t) \sigma(x(t)), \quad e(0)=e_{0},
$$

where

$$
\tilde{W}(t) \triangleq \hat{W}(t)-W \in \mathbb{R}^{(s+n) \times m},
$$

is the weight error, and $e_{0} \triangleq x_{0}-x_{\mathrm{r}_{0}}$. In addition, we write the weight error dynamics using (16) as

$$
\dot{\tilde{W}}(t)=\gamma \sigma(x(t)) e^{\mathrm{T}}(t) P B, \quad \tilde{W}(0)=\tilde{W}_{0},
$$

where $\tilde{W}_{0} \triangleq \hat{W}(0)-W$. The following lemma is now immediate.

Lemma 3.1: Consider the uncertain dynamical system given by (4) subject to (5), the reference model given by (18), and the feedback control law given by (12), (13), (15) and (16). Then, the solution $(e(t), \tilde{W}(t))$ is Lyapunov stable for all $\left(e_{0}, \tilde{W}_{0}\right) \in \mathbb{R}^{n} \times \mathbb{R}^{(s+n) \times m}$ and $t \in \overline{\mathbb{R}}_{+}$.

Proof: To show Lyapunov stability of the solution $(e(t), \tilde{W}(t))$ given by $(20)$ and $(22)$ for all $\left(e_{0}, \tilde{W}_{0}\right) \in$ $\mathbb{R}^{n} \times \mathbb{R}^{(s+n) \times m}$ and $t \in \overline{\mathbb{R}}_{+}$, consider the Lyapunov function candidate

$$
\mathcal{V}(e, \tilde{W})=e^{\mathrm{T}} P e+\gamma^{-1} \operatorname{tr}\left(\tilde{W} \Lambda^{\frac{1}{2}}\right)^{\mathrm{T}}\left(\tilde{W} \Lambda^{\frac{1}{2}}\right) .
$$

Note that $\mathcal{V}(0,0)=0, \mathcal{V}(e, \tilde{W})>0$ for all $(e, \tilde{W}) \neq$ $(0,0)$, and $\mathcal{V}(e, \tilde{W})$ is radially unbounded. Differentiating (23) along the trajectories of (20) and (22) yields

$$
\dot{\mathcal{V}}(e(t), \tilde{W}(t))=-e^{\mathrm{T}}(t) \operatorname{Re}(t) \leq 0,
$$

where the result is now immediate.

Since the solution $(e(t), \tilde{W}(t))$ is Lyapunov stable for all $\left(e_{0}, \tilde{W}_{0}\right) \in \mathbb{R}^{n} \times \mathbb{R}^{(s+n) \times m}$ and $t \in \overline{\mathbb{R}}_{+}$from Lemma 3.1, this trivially implies that $e(t) \in \mathcal{L}_{\infty}$ and $\tilde{W}(t) \in \mathcal{L}_{\infty}$. At this stage in our analysis, it should be noted that one cannot use the Barbalat's lemma (Khalil, 1996) to conclude $\lim _{t \rightarrow \infty} e(t)=0$. To elucidate this point, one can write

$$
\begin{aligned}
\ddot{\mathcal{V}}(e(t), \tilde{W}(t))= & -2 e^{\mathrm{T}}(t) R\left[A_{\mathrm{r}} e(t)\right. \\
& \left.-B \Lambda \tilde{W}^{\mathrm{T}}(t) \sigma\left(e(t)+x_{\mathrm{r}}(t)\right)\right],
\end{aligned}
$$

but since $x_{\mathrm{r}}(t)$ can be unbounded due to the coupling between the inner and outer-loop architectures, one cannot conclude the boundedness of (25), which is necessary for utilising the Barbalat's lemma in (24). Motivated from this standpoint, we next provide the conditions to ensure the boundedness of the reference model states $x_{r}(t)$, which also reveal the fundamental stability limit (FSL) for guaranteeing the closed-loop system stability. It is noted that two FSLs are provided below; namely a delay-independent FSL and a delay-dependent FSL.

\subsection{Delay-independent FSL}

A linear time-invariant system subject to time delay can in some cases be stable regardless of how large the time delay $\tau$ is (Chen \& Latchman, 1995; Gu, Kharitonov, \& Chen, 2003). This well-known delay-independent stability concept is investigated here. Mainly we present the mathematical conditions under which the system at hand can be delay-independent stable. For this, start with using (2) in (18), and first write

$$
\begin{aligned}
\dot{x}_{\mathrm{r}}(t)= & A_{\mathrm{r}} x_{\mathrm{r}}(t)+B_{\mathrm{r}}\left(C_{\mathrm{h}} \xi(t)+D_{\mathrm{h}} \theta(t-\tau)\right), \\
= & A_{\mathrm{r}} x_{\mathrm{r}}(t)-B_{\mathrm{r}} D_{\mathrm{h}} E_{\mathrm{h}} x_{\mathrm{r}}(t-\tau)+B_{\mathrm{r}} C_{\mathrm{h}} \xi(t) \\
& -B_{\mathrm{r}} D_{\mathrm{h}} E_{\mathrm{h}} e(t-\tau)+B_{\mathrm{r}} D_{\mathrm{h}} r(t-\tau) .
\end{aligned}
$$

Next, it follows from (1) that

$$
\begin{aligned}
\dot{\xi}(t)= & A_{\mathrm{h}} \xi(t)-B_{\mathrm{h}} E_{\mathrm{h}} x_{\mathrm{r}}(t-\tau)-B_{\mathrm{h}} E_{\mathrm{h}} e(t-\tau) \\
& +B_{\mathrm{h}} r(t-\tau) .
\end{aligned}
$$

Finally, by letting $\phi(t) \triangleq\left[x_{\mathrm{r}}^{\mathrm{T}}(t), \xi^{\mathrm{T}}(t)\right]^{\mathrm{T}}$, and using (26) and (27), one can write

$$
\begin{gathered}
\dot{\phi}(t)=\mathcal{A}_{0} \phi(t)+\mathcal{A}_{\tau} \phi(t-\tau)+\varphi(\cdot), \\
\phi(0)=\phi_{0},
\end{gathered}
$$

where

$$
\begin{gathered}
\mathcal{A}_{0} \triangleq\left[\begin{array}{cc}
A_{\mathrm{r}} & B_{\mathrm{r}} C_{\mathrm{h}} \\
0_{n_{\xi} \times n} & A_{\mathrm{h}}
\end{array}\right] \in \mathbb{R}^{\left(n+n_{\xi}\right) \times\left(n+n_{\xi}\right)}, \\
\mathcal{A}_{\tau} \triangleq\left[\begin{array}{cc}
-B_{\mathrm{r}} D_{\mathrm{h}} E_{\mathrm{h}} & 0_{n \times n_{\xi}} \\
-B_{\mathrm{h}} E_{\mathrm{h}} & 0_{n_{\xi} \times n_{\xi}}
\end{array}\right] \in \mathbb{R}^{\left(n+n_{\xi}\right) \times\left(n+n_{\xi}\right)}, \quad(30) \\
\varphi(\cdot) \triangleq\left[\begin{array}{c}
-B_{\mathrm{r}} D_{\mathrm{h}} E_{\mathrm{h}} e(t-\tau)+B_{\mathrm{r}} D_{\mathrm{h}} r(t-\tau) \\
-B_{\mathrm{h}} E_{\mathrm{h}} e(t-\tau)+B_{\mathrm{h}} r(t-\tau)
\end{array}\right] \in \mathbb{R}^{n+n_{\xi}} .
\end{gathered}
$$

As a consequence of Lemma 3.1 and the boundedness of the reference $r(t)$, one can conclude that $\varphi(\cdot) \in \mathcal{L}_{\infty}$. We now state the following necessary lemma for the main results of this paper. 
Lemma 3.2: Let $\mathcal{P} \in \mathbb{R}_{+}^{\left(n+n_{\xi}\right) \times\left(n+n_{\xi}\right)} \cap \mathbb{S}^{\left(n+n_{\xi}\right) \times\left(n+n_{\xi}\right)}$ and $\mathcal{S} \in \mathbb{R}_{+}^{\left(n+n_{\xi}\right) \times\left(n+n_{\xi}\right)} \cap \mathbb{S}^{\left(n+n_{\xi}\right) \times\left(n+n_{\xi}\right)}$ such that the linear matrix inequality (LMI)

$$
\mathcal{F} \triangleq\left[\begin{array}{cc}
\mathcal{A}_{0}^{\mathrm{T}} \mathcal{P}+\mathcal{P} \mathcal{A}_{0}+\mathcal{S} \mathcal{P} \mathcal{A}_{\tau} \\
\mathcal{A}_{\tau}^{\mathrm{T}} \mathcal{P} & -\mathcal{S}
\end{array}\right]<0
$$

holds. Then, $\phi(t)$ of the dynamical system given by (28) is bounded for any $\tau \in \overline{\mathbb{R}}_{+}$and for all $\phi(0) \in \mathbb{R}^{n+n_{\xi}}$ and $t \in$ $\overline{\mathbb{R}}_{+}$.

Proof: Consider the Lyapunov-Krasovskii (LK) functional candidate given by $\mathcal{V}(\phi)=\phi^{\mathrm{T}} \mathcal{P} \phi+$ $\int_{-\tau}^{0} \phi^{\mathrm{T}}(t+\mu) \mathcal{S} \phi(t+\mu) \mathrm{d} \mu$. Since $\varphi(\cdot) \in \mathcal{L}_{\infty}$, let $\varphi^{*} \in \mathbb{R}_{+}$be such that $\|\varphi(\cdot)\|_{2} \leq \varphi^{*}$. Differentiating this LK functional along the trajectory of (28) yields $\dot{\mathcal{V}}(\phi(t)) \leq \eta^{\mathrm{T}}(t) \mathcal{F} \eta(t)+2 \lambda_{\max }(\mathcal{P}) \varphi^{*}\|\eta(t)\|_{2}$, where $\eta(t) \triangleq\left[\phi^{\mathrm{T}}(t), \phi^{\mathrm{T}}(t-\tau)\right]^{\mathrm{T}}$. If (32) holds, it then follows that $\dot{\mathcal{V}}(\phi(t)) \leq-k\|\eta(t)\|_{2}\left(\|\eta(t)\|_{2}-2 k^{-1} \lambda_{\max }(\mathcal{P}) \varphi^{*}\right)$, where $k \triangleq-\lambda_{\min }(\mathcal{F})$. Consequently, there exists a compact set $\mathcal{R} \triangleq\left\{\eta(t) \in \mathbb{R}^{2\left(n+n_{\xi}\right)}: \quad\|\eta(t)\|_{2} \leq\right.$ $\left.2 k^{-1} \lambda_{\max }(\mathcal{P}) \varphi^{*}\right\}$ such that $\dot{\mathcal{V}}(\phi(t))<0$ outside of this set, which proves the boundedness of (28) for any $\tau \in \overline{\mathbb{R}}_{+}$and for all $\phi(0) \in \mathbb{R}^{n+n_{\xi}}$ and $t \in \overline{\mathbb{R}}_{+}$.

Note that the LMI given by (32) is standard in the literature (for example, see Theorem 3.1 of Verriest and Ivanov (1994) for the same LMI appearing when $\varphi(\cdot) \equiv$ $0)$. Lemma 3.2 establishes the boundedness of not only the reference model states, the dynamics of which are given by (18), but also the internal human dynamics given by (1), and hence, $x_{\mathrm{r}}(t) \in \mathcal{L}_{\infty}$ and $\xi(t) \in \mathcal{L}_{\infty}$. We are now ready to state the first main result of this paper.

Theorem 3.1: Consider the uncertain dynamical system given by (4) subject to (5), the reference model given by (18), the feedback control law given by (12), (13), (15) and (16), and the human dynamics given by (1)-(3). Then, $e(t) \in \mathcal{L}_{\infty}$ and $\tilde{W}(t) \in \mathcal{L}_{\infty}$. If, in addition, there exist $\mathcal{P} \in$ $\mathbb{R}_{+}^{\left(n+n_{\xi}\right) \times\left(n+n_{\xi}\right)} \cap \mathbb{S}^{\left(n+n_{\xi}\right) \times\left(n+n_{\xi}\right)}$ and $\mathcal{S} \in \mathbb{R}_{+}^{\left(n+n_{\xi}\right) \times\left(n+n_{\xi}\right)} \cap$ $\mathbb{S}^{\left(n+n_{\xi}\right) \times\left(n+n_{\xi}\right)}$ such that the LMI given by (32) holds, then $x_{\mathrm{r}}(t) \in \mathcal{L}_{\infty}, \xi(t) \in \mathcal{L}_{\infty}$ and $\lim _{t \rightarrow \infty} e(t)=0$.

Proof: As a consequence of Lemma 3.1, recall that $e(t) \in \mathcal{L}_{\infty}$ and $\tilde{W}(t) \in \mathcal{L}_{\infty}$. In addition, note that $\varphi(\cdot) \in$ $\mathcal{L}_{\infty}$ in (28). Next, if there exist $\mathcal{P} \in \mathbb{R}_{+}^{\left(n+n_{\xi}\right) \times\left(n+n_{\xi}\right)} \cap$ $\mathbb{S}^{\left(n+n_{\xi}\right) \times\left(n+n_{\xi}\right)}$ and $\mathcal{S} \in \mathbb{R}_{+}^{\left(n+n_{\xi}\right) \times\left(n+n_{\xi}\right)} \cap \mathbb{S}^{\left(n+n_{\xi}\right) \times\left(n+n_{\xi}\right)}$ such that the LMI given by (32) holds, recall from Lemma 3.2 that $x_{\mathrm{r}}(t) \in \mathcal{L}_{\infty}$ and $\xi(t) \in \mathcal{L}_{\infty}$. Finally, since $e(t) \in$ $\mathcal{L}_{\infty}, x_{\mathrm{r}}(t) \in \mathcal{L}_{\infty}$ and $\tilde{W}(t) \in \mathcal{L}_{\infty}$ ensure the boundedness of (25), it now follows from the Barbalat's lemma that $\lim _{t \rightarrow \infty} e(t)=0$.

For the boundedness of all closed-loop system signals and $\lim _{t \rightarrow \infty} e(t)=0$, Theorem 3.1 requires the FSL given by the LMI (32) to hold. Note that this FSL can be equivalently written in an equality form as Richard (2003)

$$
0=\mathcal{A}_{0}^{\mathrm{T}} \mathcal{P}+\mathcal{P} \mathcal{A}_{0}+\mathcal{P} \mathcal{A}_{\tau} \mathcal{S}^{-1} \mathcal{A}_{\tau}^{\mathrm{T}} \mathcal{P}+\mathcal{S}+\mathcal{Q},
$$

where $\quad \mathcal{P} \in \mathbb{R}_{+}^{\left(n+n_{\xi}\right) \times\left(n+n_{\xi}\right)} \cap \mathbb{S}^{\left(n+n_{\xi}\right) \times\left(n+n_{\xi}\right)}, \quad \mathcal{S} \in$ $\mathbb{R}_{+}^{\left(n+n_{\xi}\right) \times\left(n+n_{\xi}\right)} \cap \mathbb{S}^{\left(n+n_{\xi}\right) \times\left(n+n_{\xi}\right)}$, and $\mathcal{Q} \in \mathbb{R}_{+}^{\left(n+n_{\xi}\right) \times\left(n+n_{\xi}\right)}$ $\cap \mathbb{S}^{\left(n+n_{\xi}\right) \times\left(n+n_{\xi}\right)}$ with $\mathcal{A}_{0}$ and $\mathcal{A}_{\tau}$, respectively, given by (29) and (30). Importantly, in addition, note that $\mathcal{A}_{0}$ and $\mathcal{A}_{\tau}$ do not depend on any unknown parameters and they only depend on the given set of human model and reference model parameters. As a consequence, for a given human model of the form (1)-(3), if the FSL respects (33) (or, equivalently (32)) with respect to a judiciously chosen reference model parameters, then the trajectories of the nonlinear closed-loop system including uncertainties and controlled by by MRAC are guaranteed to be stable.

Notice that the proof of delay-independent stability in Lemma 3.2 is based on a time-domain technique using a Lyapunov-Krasovskii functional, also see Gu et al. (2003). A large body of literature was devoted to this effort where one main focus was to reduce the inherent conservatism imposed by the choice of candidate functionals. An alternative 'matrix measure method' is proposed in Nguyen and Summers (2011) to this end. Another method would be to employ frequency-domain tools where one instead studies the eigenvalues of the corresponding linear timeinvariant system with time delay. For example, consider the nominal part of (28); e.g. $\varphi(\cdot) \equiv 0$ and let $\tau \rightarrow \infty$. In this case, the system will behave like an open-loop system whose stability is determined by the eigenvalues of $\mathcal{A}_{0}$. For the system to be stable in the open-loop setting, $\mathcal{A}_{0}$ must be Hurwitz. That is, the stability of the open-loop system is a necessary condition for delay-independent stability. Next, note that matrix $\mathcal{A}_{0}$ is invertible since it is Hurwitz. Hence, the characteristic function of the dynamical system

$$
f:=\operatorname{det}\left[s I-\mathcal{A}_{0}-\mathcal{A}_{\tau} e^{-s \tau}\right]
$$

can be rearranged as

$$
\operatorname{det}\left[I-\left(s I-\mathcal{A}_{0}\right)^{-1} \mathcal{A}_{\tau} e^{-s \tau}\right] \cdot \operatorname{det}\left[s I-\mathcal{A}_{0}\right] .
$$

Note that for the class of time-delay systems being considered here, as a parameter of interest; e.g. delay, changes, the system may switch from a stable to unstable regime (or vice versa) only if the system has imaginary eigenvalues $s=j \omega$ (Stépán, 1989). Investigation of whether or not such a switch could arise then requires studying the zeros of the system characteristic function (35) at $s=j \omega$, where $\omega \geq 0$ without loss of generality. On the 
imaginary axis, however, only the first determinant in (35) can be zero since the second determinant is always non-zero owing to $\mathcal{A}_{0}$ being Hurwitz. One can then follow a number of techniques to test whether or not the dynamics is delay-independent stable. One practical and widely utilised technique is the one proposed in Chen and Latchman (1995), which requires simple computations to assess delay-independent stability, as presented next.

Corollary 3.1: Let the human dynamics given by (1)-(3) be a single-input-single-output system (SISO) with gain $k_{p}$. Then, for (28) with $\varphi(\cdot) \equiv 0$ to be delay-independent stable, it is necessary that

$$
k_{p}<\frac{1}{\rho\left(A_{\mathrm{r}}^{-1} B_{\mathrm{r}} E_{\mathrm{h}}\right)}
$$

holds, where $\rho(\cdot)$ denotes the spectral radius.

Proof: Start with (29) and (30) and rewrite the characteristic function (34) explicitly as

$f:=\operatorname{det}\left[s I-A_{\mathrm{r}}+B_{\mathrm{r}}\left(C_{\mathrm{h}}\left(s I-A_{\mathrm{h}}\right)^{-1} B_{\mathrm{h}}+D_{\mathrm{h}}\right) E_{\mathrm{h}} e^{-\tau s}\right]$,

which simplifies to

$$
f:=\operatorname{det}\left[s I-A_{\mathrm{r}}+B_{\mathrm{r}} E_{\mathrm{h}} G(s) e^{-\tau s}\right],
$$

where $G(s)$ is the scalar transfer function corresponding to the SISO system given by (1) and (2). Note that the above expression is in the exact form as (34) hence for (28) with $\varphi(\cdot) \equiv 0$ to be delay-independent stable, it is necessary that the open-loop system is stable, which requires that $A_{\mathrm{r}}$ is Hurwitz. As per the construction in (13), this always holds. Then, from Chen and Latchman (1995), it is also necessary that stability is guaranteed for $s=0$. This condition is equivalent to

$$
\rho\left(\left(-A_{\mathrm{r}}\right)^{-1}\left(B_{\mathrm{r}} E_{\mathrm{h}}\right) k_{p}\right)<1,
$$

where we made the substitution $k_{p}=G(0)$. This then gives (36), and hence, the proof is complete.

It is worthy to note that the results in Corollary 3.1 can be further improved in many practical scenarios. For example, observe that the reference input to the human model and the human command are of dimension one in the SISO case. In addition, since generally the outer-loop and inner-loop command following objectives are the same, note that $E_{\mathrm{h}_{\mathrm{p}}}=E_{\mathrm{p}}$ (see also the illustrative numerical example given in Section 4). Thus, in view of these, the following result is now immediate.
Corollary 3.2: Given $E_{\mathrm{h}_{\mathrm{p}}}=E_{\mathrm{p}}$ and under the conditions in Corollary 3.1, the necessary condition for the humanin-the loop MRAC model (28) with $\varphi(\cdot) \equiv 0$ to be delayindependent stable is given by

$$
k_{p}<1
$$

Proof: Note that $A_{\mathrm{r}}^{-1} B_{\mathrm{r}}$ and $E_{\mathrm{h}}$ in (36) are column vectors. Therefore, as per Appendix 1, we have $\rho\left(A_{\mathrm{r}}^{-1} B_{\mathrm{r}} E_{\mathrm{h}}\right)=\left|E_{\mathrm{h}} A_{\mathrm{r}}^{-1} B_{\mathrm{r}}\right|$. Since in the scalar case $E_{\mathrm{h}} A_{\mathrm{r}}^{-1} B_{\mathrm{r}}=-1$ as per Appendix 2, then (40) follows.

In the above corollary, we prove that the human gain must be less than one such that (28) with $\varphi(\cdot) \equiv 0$ can have a chance to be delay-independent stable. Sufficiency can be numerically studied by checking whether or not $\rho\left(\left(j \omega-A_{\mathrm{r}}\right)^{-1}\left(B_{\mathrm{r}} E_{\mathrm{h}}\right) G(j \omega)\right)$ is less than one for the bounded single sweep parameter $\omega \geq 0$, see Chen and Latchman (1995) as well as the next section. What is interesting in the above analysis is that human's aggressiveness as measured by $k_{p}$ can be a strong limiting factor ruining delay-independent stability. Moreover, since by the design of stable MRAC we have zero steady-state error in tracking, the necessary condition $k_{p}<1$ is solely inherent to the human's gain and holds irrespective of the model-reference controller gain $K$. While in many cases it is reasonable to assume that the human model can be considered as SISO dynamics; e.g. when the human produces a single output to steer a manipulator, in the case when an auto-human model is utilised in multi-inputmulti-output (MIMO) settings, the necessary condition (39) can be revised as follows:

$$
\rho\left(A_{\mathrm{r}}^{-1} B_{\mathrm{r}}[G(0)] E_{\mathrm{h}}\right)<1,
$$

where $[G(0)]$ denotes the matrix transfer function of the MIMO auto-human model with $s=0$ in all its entries.

It is important to note that while guaranteeing delayindependent stability in a dynamical system is attractive as this makes the system completely immune to destabilising effects of delays, in some cases by the nature of the problem, delay-independent stability cannot be possible as is the case above for $k_{p}>1$. Indeed, in the case when MRAC deals with a relatively more aggressive human behaviour with $k_{p}>1$, it is impossible to avoid instability for some delay values $\tau$. One then wonders how large these delays can be before instability is introduced. Moreover, a trade-off in delay-independent stable cases arises in particular on system's performance, which may deteriorate for large delays despite stability is preserved (Nia \& Sipahi, 2014). In light of this, we now turn our attention to the case when delay-independent stability is not possible, or not desired, and hence, system stability is affected by the numerical value of the delay in the dynamical system. 


\subsection{Delay-dependent FSL}

Delay-independent FSL given in the previous section guarantees the boundedness of all closed-loop system signals and $\lim _{t \rightarrow \infty} e(t)=0$ for any $\tau \in \overline{\mathbb{R}}_{+}$. Since the time delay in human dynamics can in general be known in practice for certain applications, at least within a certain range, it is possible to relax these conditions by utilising the delay information in the stability analysis. Towards this goal, we first provide the following lemma.

Lemma 3.3: Consider the following system dynamics given by

$$
\dot{z}(t)=F z(t)+G z(t-\tau)+h(t, z(t)), \quad z(0)=z_{0},
$$

where $z(t) \in \mathbb{R}^{n}$ is the state vector, $F \in \mathbb{R}^{n x n}$ and $G \in \mathbb{R}^{n x n}$ are constant matrices, $\tau$ is the time delay, and $h(t, z(t))$ is piecewise continuous and bounded nonlinear forcing term, which is in general a function of state $z$. If the homogeneous dynamical system given by

$$
\dot{z}(t)=F z(t)+G z(t-\tau),
$$

is asymptotically stable, then the states of the original inhomogeneous dynamical system given by (42) remain bounded for all times.

Proof: Since $h(t, z(t))$ is piecewise continuous and bounded, this signal can be considered as an exogenous input to the homogeneous system (43). Under the assumption that this system is asymptotically stable, the output $z(t)$ of (42) remains bounded.

Having established Lemma 3.3, we are now ready to state the second main result of this paper, which provides a more relaxed delay-dependent stability condition for the overall human-in-the-loop system and convergence of the system error, $e(t)$, to zero.

Theorem 3.2: Consider the uncertain dynamical system given by (4) subject to (5), the reference model given by (18), the feedback control law given by (12), (13), (15) and (16), and the human dynamics given by (1)-(3). Then, $e(t) \in \mathcal{L}_{\infty}$ and $\tilde{W}(t) \in \mathcal{L}_{\infty}$. If, in addition, the real parts of all the infinitely many roots of the following characteristic equation

$$
\operatorname{det}\left(s I-\left(\mathcal{A}_{0}+\mathcal{A}_{\tau} e^{-\tau s}\right)\right)=0
$$

have strictly negative real parts, then $x_{\mathrm{r}}(t) \in \mathcal{L}_{\infty}, \xi(t) \in$ $\mathcal{L}_{\infty}$, and $\lim _{t \rightarrow \infty} e(t)=0$.

Proof: As a consequence of Lemma 3.1, recall that $e(t) \in$ $\mathcal{L}_{\infty}$ and $\tilde{W}(t) \in \mathcal{L}_{\infty}$. In addition, note that $\varphi(\cdot) \in \mathcal{L}_{\infty}$ in (28). Therefore, if all of the roots of the characteristic equation given by (44) have strictly negative real parts, making the homogeneous equation

$$
\dot{\phi}(t)=\mathcal{A}_{0} \phi(t)+\mathcal{A}_{\tau} \phi(t-\tau)
$$

asymptotically stable, then, as per Lemma 3.3, $\phi(t) \triangleq\left[x_{\mathrm{r}}^{\mathrm{T}}(t), \xi^{\mathrm{T}}(t)\right]^{\mathrm{T}} \in \mathcal{L}_{\infty}$. Finally, since $e(t) \in \mathcal{L}_{\infty}$, $x_{\mathrm{r}}(t) \in \mathcal{L}_{\infty}$, and $\tilde{W}(t) \in \mathcal{L}_{\infty}$ ensure the boundedness of (25), it now follows from the Barbalat's lemma that $\lim _{t \rightarrow \infty} e(t)=0$.

Remark 3.1: Several methods are available in the literature for the analysis of the root locations of (44). The four most used methods are TRACE-DDE (Breda, Maset, \& Vermiglio, 2006), DDE-BIFTOOL (Engelborghs, Luzyanina, \& Roose, 2000), QPMR Vyhlidal and Zitek (2009) and Lambert-W function (Yi, Nelson, \& Ulsoy, 2010). In essence, one provides the matrices $\mathcal{A}_{0}$ and $\mathcal{A}_{\tau}$ as well as the delay $\tau$ to these methods and obtains the numerical values of the rightmost root locations of (44). In some sense, these methods perform a non-trivial approximation of the infinite-dimensional spectrum of the system (45) with which they are able to identify the most relevant roots - the rightmost roots. In the illustrative numerical example provided below, we employ TRACE-DDE (available for downloading Breda, 2008).

Before we close this section, we remark that the above-presented stability analysis approach, for both delay-dependent and delay-independent cases, consists of studying two interconnected subsystems $\mathcal{S}_{1}$ and $\mathcal{S}_{2}$ in a sequential manner, where $\mathcal{S}_{1}$ refers to the inner-loop system while $\mathcal{S}_{2}$ refers to the outer-loop system, which comprises the model reference dynamics and the homogeneous linear time-invariant system modelling the human dynamics. It is important to realise that the inner-loop system $\mathcal{S}_{1}$ is delay free but the outer-loop system is influenced by human reaction delay $\tau$. To be able to prove the overall stability of the combined systems $\mathcal{S}_{1}$ and $\mathcal{S}_{2}$, we first start only with $\mathcal{S}_{1}$, and show the boundedness of the signals in the inner loop consisting of the reference model, controller, uncertain dynamical system and the parameter adjustment mechanism (see Figure 1). This is achieved through Lemma 3.1 concerning the stability of the tracking error $e(t)$ and adaptive control parameter vector $\hat{W}(t)$ dynamics. Next, Lemma 3.2 proves the boundedness of the internal human dynamics states $\xi(t)$ in $\mathcal{S}_{2}$ as well as the reference model states $x_{r}(t)$. Theorem 3.1 then proves the asymptotic stability of the innerloop system $\mathcal{S}_{1}$ and the delay-independent stability of the overall closed-loop dynamics $\mathcal{S}_{1}$ and $\mathcal{S}_{2}$ combined. A similar procedure is followed for the delay-dependent case, where Lemma 3.1 and Theorem 3.1 are replaced, respectively, by Lemma 3.3 and Theorem 3.2. 
Ultimately, the above-developed framework enables that the stability of the nonlinear time-delayed system can be proved by the decomposition of the total system into two subsystems $\mathcal{S}_{1}$ and $\mathcal{S}_{2}$, namely the inner and outer loops, and by interconnecting the stability proofs obtained independently for each subsystem. To the best knowledge of the authors, this is a new development and philosophy that can be useful in analysing and designing coupled inner-outer loop systems. Specifically, the framework provides a constructive way to treat the problem in sequential steps and lays out tools to design the controllers, which can be easier than treating the entire problem all at once.

While different from this framework, notable results were reported on the stability of nonlinear systems with state delays, for example, see input-to-state stability (ISS) and integral-ISS (iISS) methodology in Sontag (1998) and Pepe and Jiang $(2005,2006)$. Interestingly, in Pepe and Jiang (2006, p. 4209), the authors point out the opportunity of utilising delay-dependent and delay-independent stability conditions of linear systems with delays towards studying iISS properties of bilinear systems with delays using a carefully selected Lyapunov-Krasovskii functional. Although this development is quite different than the one presented above, its philosophy is aligned with ours; that is, there is the strong potential to treat nonlinear systems with time delays in modular ways while benefitting from existing theories on linear systems with time delays.

\section{Illustrative numerical example}

Consider the longitudinal motion of a Boeing 747 airplane linearised at an altitude of $40 \mathrm{kft}$ and a velocity of $774 \mathrm{ft} / \mathrm{sec}$ with the dynamics given by Bryson (1994)

$\dot{x}(t)=A_{p} x(t)+B_{p}\left(u(t)+W^{\mathrm{T}} \sigma(x(t)), \quad x(0)=x_{0}\right.$,

where $x(t)=\left[x_{1}(t), x_{2}(t), x_{3}(t), x_{4}(t)\right]^{\mathrm{T}}$ is the state vector. Note that (46) can be equivalently written as (4) with $\Lambda=I$. Here, $x_{1}(t), x_{2}(t)$ and $x_{3}(t)$, respectively, represent the components of the velocity along the $x, z$ and $y$ axes of the aircraft with respect to the reference axes (in $\mathrm{crad} / \mathrm{sec}$ ), $x_{4}(t)$ represents the pitch Euler angle of the aircraft body axis with respect to the reference axes (in crad), and $u(t) \in \mathbb{R}$ represents the elevator control input (in crad), where 0.01 radian $=1 \mathrm{crad}$ (centiradian). Finally, $W \in \mathbb{R}^{3}$ is an unknown weighting matrix and $\sigma(x(t))=\left[1, x_{1}(t), x_{2}(t)\right]^{\mathrm{T}}$ is a known basis function. In the following simulations, unless stated otherwise, we set $W=\left[\begin{array}{lll}0.1 & 0.3 & -0.3\end{array}\right]^{T}$. The dynamical system given in
Table 1. Numerical data used in the illustrative numerical example.

\begin{tabular}{|c|c|}
\hline$T$ & 1 \\
\hline$T_{7}^{p}$ & 5 \\
\hline$\tau$ & 0.5 \\
\hline$A_{\mathrm{p}}$ & $\begin{array}{c}{[-0.0030 .0390-0.322 ;-0.065-} \\
0.3197 .7400 ; 0.020-0.101-0.4290 ; 0010]\end{array}$ \\
\hline$B_{\mathrm{p}}$ & {$[0.010 ;-0.180 ;-1.160 ; 0]$} \\
\hline$E_{p}^{\mathrm{p}}$ & {$\left[\begin{array}{llll}0 & 0 & 0 & 1\end{array}\right]$} \\
\hline$E_{h}$ & {$\left[\begin{array}{lllll}0 & 0 & 0 & 1 & 0\end{array}\right]$} \\
\hline$B_{r}^{\prime \prime}$ & {$\left[\begin{array}{lllll}0 & 0 & 0 & 0 & 1\end{array}\right]^{\top}$} \\
\hline$Q$ & $\operatorname{diag}\left(\left[\begin{array}{lllll}0 & 0 & 0 & 1 & 2.5\end{array}\right]\right)$ \\
\hline
\end{tabular}

(46) is assumed to be controlled using a model reference adaptive controller, the details of which are explained in Section 2. In addition, the aircraft is assumed to be operated by a pilot whose Neal-Schmidt Model (Schmidt \& Bacon, 1983) is given by

$$
k_{p} \frac{T_{p} s+1}{T_{z} s+1} e^{-\tau s},
$$

where $k_{p}$ is the positive scalar pilot gain, $T_{p}$ and $T_{z}$ are positive scalar time constants, and $\tau$ is the pilot reaction time delay. Parameter values used in the simulations are provided in Table 1.

To obtain the nominal controller $K$, a linear quadratic regulator (LQR) approach is utilised with the following objective function to be minimised:

$$
J(\cdot)=\int_{0}^{\infty}\left(x^{\mathrm{T}}(t) Q x(t)+\mu u^{2}(t)\right) \mathrm{d} t
$$

where $Q$ is a positive-definite weighting matrix of appropriate dimension and $\mu$ is a positive weighting scalar. Notice that the framework developed in Section 2 is not limited to a particular design method for the nominal controller. To this end, this task can be handled by a number of different ways. Here LQR is utilised for convenience reasons. In this setting, the selection of the weighing matrices, as expected, will affect the resulting nominal controller gain $K$ in (13), which in turn will determine the reference model dynamics (18). In the following simulation studies, the effect of the weighting matrices, and thus the effects of reference model parameters on the overall closed-loop system stability are investigated for various values of pilot model parameters. To facilitate the analysis, reference model parameter variations are achieved mainly by manipulating the control penalty variable $\mu$.

Note that the purpose of the numerical examples provided in this section is to verify the theoretical stability predictions of the proposed framework. Therefore, the simulation results are created to present the stability/instability of the closed-loop system without further 
tuning parameters for enhanced transient response characteristics.

\subsection{Delay-independent stability}

\subsubsection{LMI approach}

We set $k_{p}=1 / 2$ and investigate whether or not the nominal part of the closed-loop system in (28) is delayindependent stable. Specifically, we first use the LQR control designer in MATLAB with $\mu=1.0$ to design $K$, which returns $K=[-0.0185,0.0815,-1.5809,-2.7560$, -1.5811]. Next, the matrices $A_{0}$ and $A_{\tau}$ are constructed based on the information provided in Table 1. Assigning $\mathcal{P}$ and $\mathcal{S}$ as positive definite, greater than $0.5 I \in$ $\mathbb{R}^{\left(n+n_{\xi}\right) \times\left(n+n_{\xi}\right)}$ while imposing the negativity constraint in (32) as $\mathcal{F}<-0.1 I \in \mathbb{R}^{\left(n+n_{\xi}\right) \times\left(n+n_{\xi}\right)}$, the YALMIP LMI optimisation toolbox returns a feasible set of matrices $\mathcal{P}$ and $\mathcal{S}$, indicating that the closed-loop system is delayindependent stable.

\subsubsection{Frequency-domain approach}

To be consistent with the previous subsection, we set $k_{p}=1 / 2$ and $\mu=1.0$ in the LQR optimisation. Based on Corollary 3.2, since $k_{p}<1$ and $A_{\mathrm{r}}$ is Hurwitz, the necessary conditions for delay-independent stability are satisfied. Next, the sufficient condition is studied simply by checking whether or not the metric $\mathcal{M}(\omega):=\rho((j \omega-$ $\left.\left.A_{\mathrm{r}}\right)^{-1}\left(B_{\mathrm{r}} E_{\mathrm{h}}\right) G(j \omega)\right)$ is less than one for $\omega \geq 0$, see details in Chen and Latchman (1995). We find out that the metric value $\mathcal{M}(0)=k_{p}=1 / 2$ decreases for larger $\omega \neq 0$, while remaining always less than 1 . That is, the nominal part of the closed-loop system (28) will remain stable for any choice of delay $\tau$. Keeping $\mu=1$ but letting $k_{p}=0.95$ has only negligible effects on $K$, and again it is easy to show that $\mathcal{M}(\omega)<1$ for $\omega \geq 0$. On the other hand, selecting $k_{p}=1.05$ violates this condition, that is, the system loses its delay-independent stability characteristics.

\subsection{Delay-dependent stability}

\subsubsection{Effect of control penalty on system stability for different pilot reaction time delays}

To investigate the effects of the reference model parameter variations on the stability of the closed-loop system, the control weight $\mu$ is manipulated by assigning values in the range $[0,50]$. Then, the real part of the rightmost pole (RMP) of the system, whose characteristic equation is given by (44), is plotted against these $\mu$ values. This procedure is repeated for various pilot reaction time delays and the results are presented in Figure 2.

Figure 2 reveals several interesting results. First, it is shown that if the reference model dynamics is not designed carefully with an appropriate $\mu$ value, then the human-in-the-loop adaptive control system can be indeed unstable as characterised by the instability (RMP $>0)$ of the nominal linear time-invariant dynamics with delay in (28). Second, this linear system can be stable for small and large values of the parameter $\mu$ and be unstable in between. Third, it is observed that as the pilot reaction time delay increases, the unstable region of $\mu$ gets larger as indicated by RMP $>0$. Ultimately, these stability/instability characteristics will be reflected to the closed-loop dynamics with uncertainties and controlled with MRAC, as per the results established in the previous section.

Consider next in Figure 2 the case for $\mu=10$, where pilot reaction time delay $\tau=0.2$ and $\tau=0.5$ result, respectively, in a stable and unstable linear time-invariant system with delay in (28). Time-domain tracking and

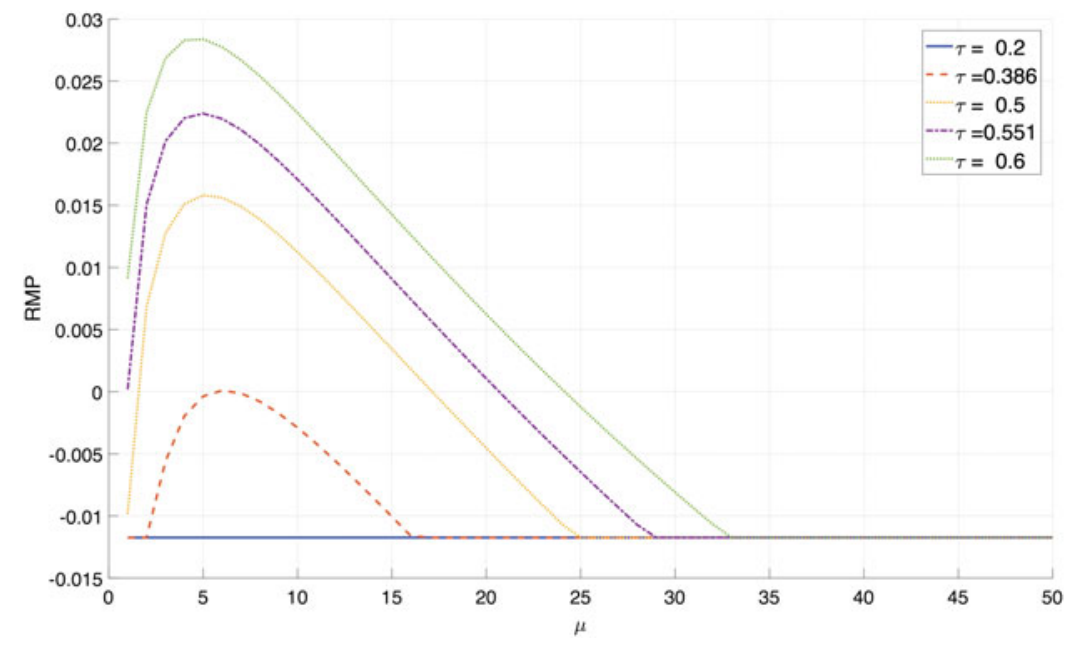

Figure 2. The real part of the rightmost pole (RMP) of (45) with respect to the control penalty variable $\mu$, for different pilot reaction time delays. 

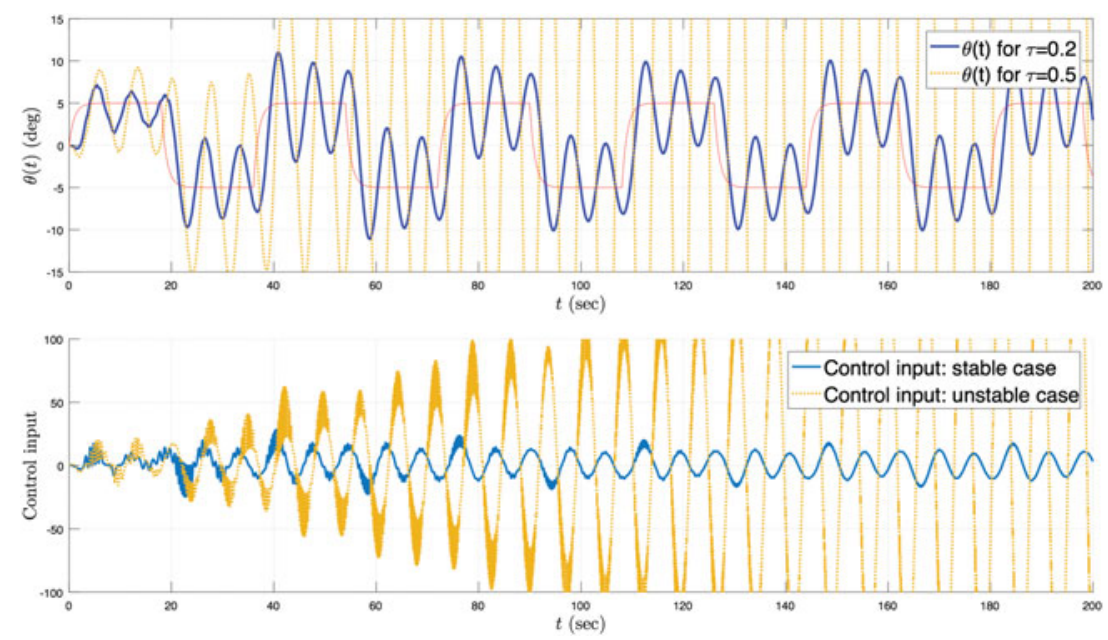

Figure 3. Tracking and control signal curves under uncertainties and MRAC for two different values of the pilot reaction time delays, $\tau=$ 0.2 and $\tau=0.5$, with $\mu=10$ in the LQR design.

control signal plots corresponding to the closed-loop system with uncertainties and controlled by MRAC presented in Figure 3 confirm this prediction. As noted earlier, the simulation results are employed to verify the theoretical stability predictions of the proposed method and therefore controllers are not tuned to obtain the best transient response. This investigation is left to future research.

It is noted that the numerical value of the considered uncertainty weighting matrix does not affect the stability of the system, mainly because the fundamental stability theorems developed in the previous sections do not pose any restrictions on the amount of uncertainty. However, it is expected that the amount of uncertainty will affect the performance of the controllers. To this end, Figure 4 is provided where the tracking and control signal curves are compared for two different values of uncertain weighting matrix, one with $W_{1}:=W=[0.1,0.3,-0.3]^{T}$ originally used for plotting Figure 3 and the other $W_{2}=2 W_{1}$ representing increased uncertainty due to doubling of $W_{1}$. As seen in Figure 4, although tracking performance remains similar in both cases, control signal amplifies in the second case to be able to accommodate the increased uncertainty in the closed-loop system.

\subsubsection{Effect of control penalty on system stability for different values of pilot model poles}

The poles of the pilot model (47) represent how fast the pilot responds to changes in the aircraft pitch angle, which can also be interpreted as pilot aggressiveness. In this
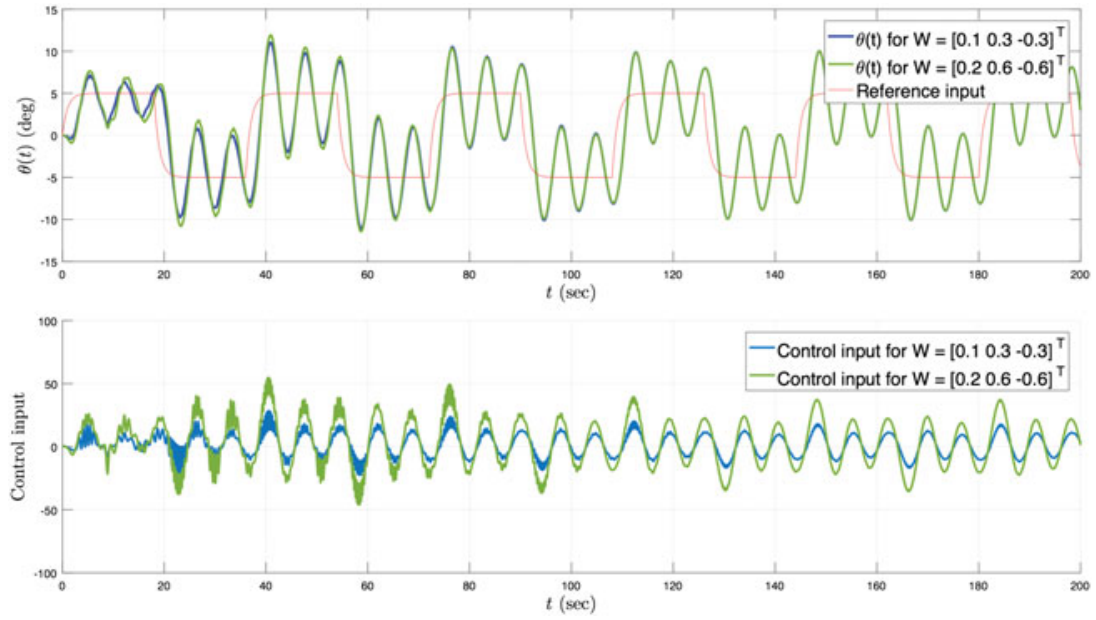

Figure 4. Tracking and control signal curves under uncertainties and MRAC for two different values of the considered weighting matrix: $W=[0.1,0.3,-0.3]^{T}$ (the value considered for plotting Figure 3 ) and $W=[0.2,0.6,-0.6]^{T}$. 


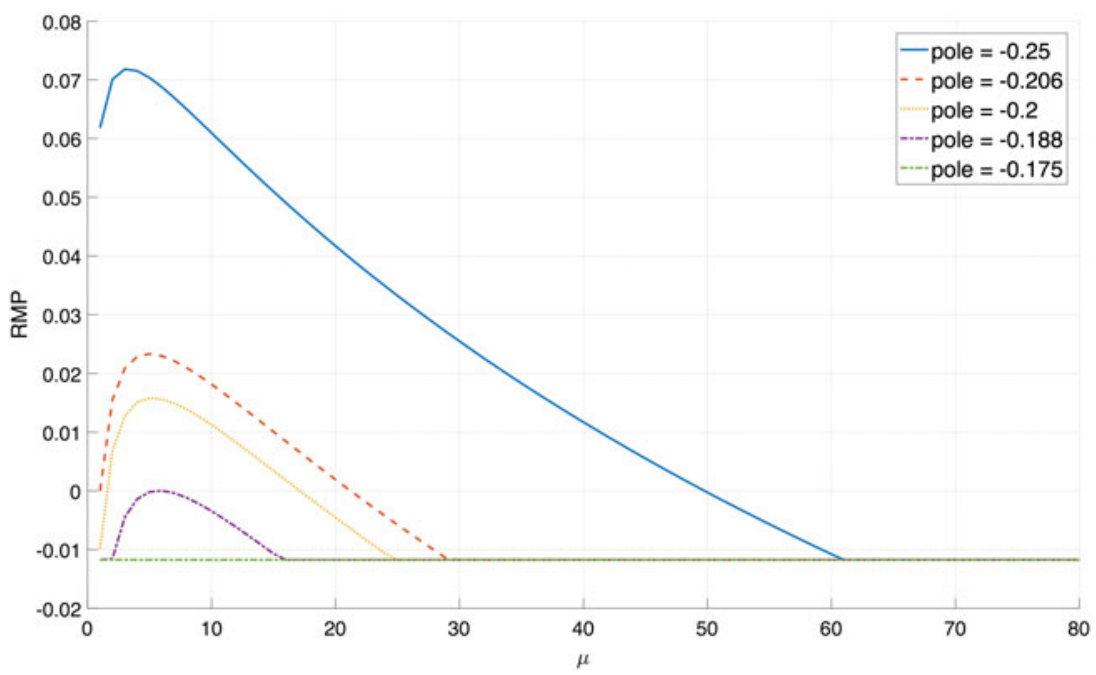

Figure 5. The location of the right most pole of (45) with respect to the control penalty variable $\mu$, for different pilot transfer function pole locations.

section, the effect of pilot aggressiveness on system stability is investigated while assigning values to the control penalty $\mu$ from 0 to 50 .

Figure 5 depicts the effect of the pilot pole locations on the real part of the RMP of the linear time-invariant dynamics with delay. The zero location and the time delay of the pilot model are kept at their nominal values of -1 and 0.5 , respectively. It is seen from the figure that, in general, unstable-stable-unstable transition is observed for increasing values of $\mu$ and, as expected, higher values of poles, corresponding to faster pilot response, decrease the $\mu$ region of stability.

Figure 6 depicts how tracking and control signal curves of closed-loop dynamics with uncertainties and controlled by MRAC are impacted by linear pilot model with two different pole locations; i.e. -0.175 and -0.2 , when $\mu=10$. As predicted in Figure 5, closed-loop system remains stable when the pole is located at -0.175 and becomes unstable when the pole is at -0.2 .

\subsubsection{Effect of control penalty on system stability for different values of pilot model zeros}

In this section, the effect of zeros of the linear pilot transfer function (47) on the stability of the overall closed-loop dynamics with uncertainties and controlled by MRAC is investigated where the control penalty $\mu$ takes values in the range $[0,50]$. The pole location and the time delay of the pilot transfer function are kept at their nominal values of -0.2 and 0.5 , respectively. Changes in the zero location of the model can be interpreted as an adjustment to the
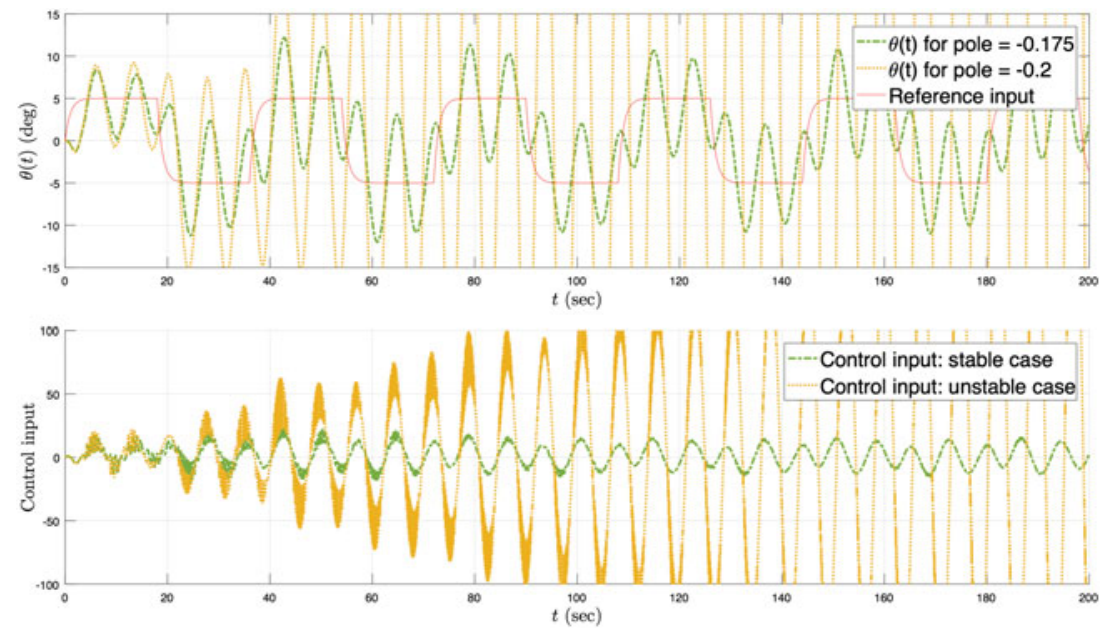

Figure 6. Tracking and control signal curves under uncertainties and MRAC for two different values of the pilot transfer function pole locations, $p=-0.175$ and $p=-0.2$, when $\mu=10$. 


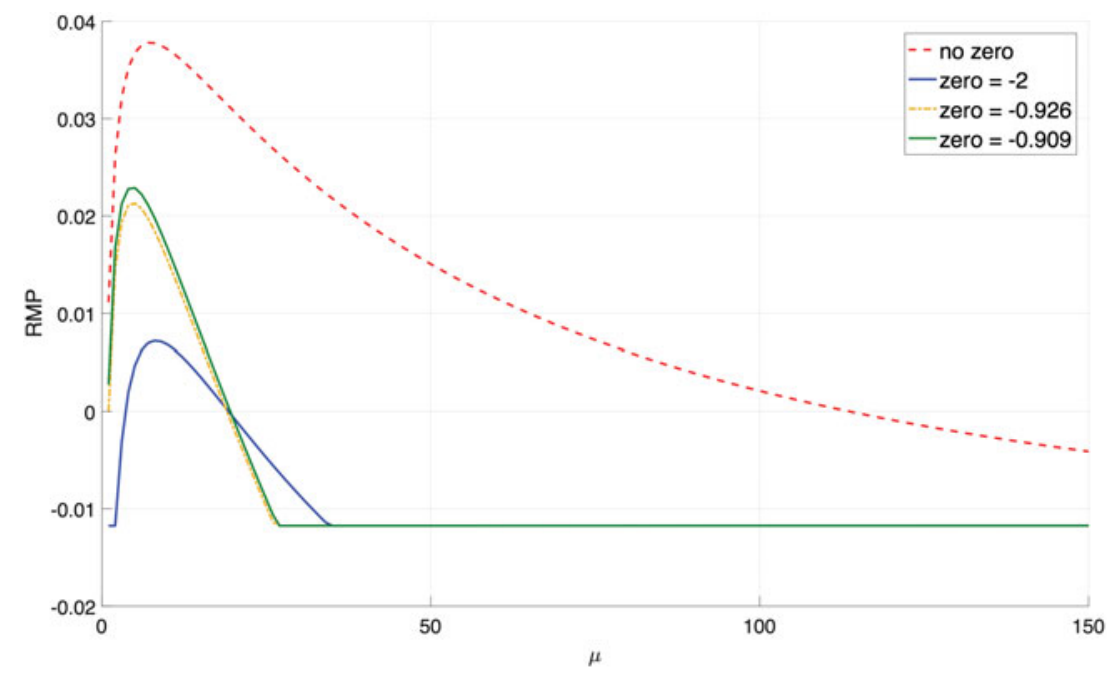

Figure 7. The location of the rightmost pole of (45) with respect to the control penalty variable $\mu$, for different pilot transfer function zero locations.

'lead' nature of the pilot, which is related to pilot's anticipation capabilities.

As seen in Figure 7, stable-unstable-stable transition structure still exists, in general, for increasing $\mu$ values. Furthermore, it is seen that when the pilot transfer function does not have a zero, a large $\mu$ region of instability arises. It is noted that for the given nominal values of the system parameters, no value of zero can make the system always stable, regardless of the considered range of $\mu$. This is mainly because delayindependent stability is determined only by the pilot's gain $k_{p}$, as per the results established in the previous section.
Figure 8 presents tracking and control signal curves for pilot model zero locations -0.2 and -0.909 , for the case when $\mu=1$. As predicted in Figure 7, the closed-loop system becomes stable for the former but unstable for the latter zero value.

\subsubsection{Effect of control penalty on system stability for different values of pilot model gains}

The pilot gain $k_{p}$ in (47) determines the intensity of the response that the pilot gives to the pitch angle deviations in the aircraft. In some sense, this gain also represents the aggressiveness of the pilot.
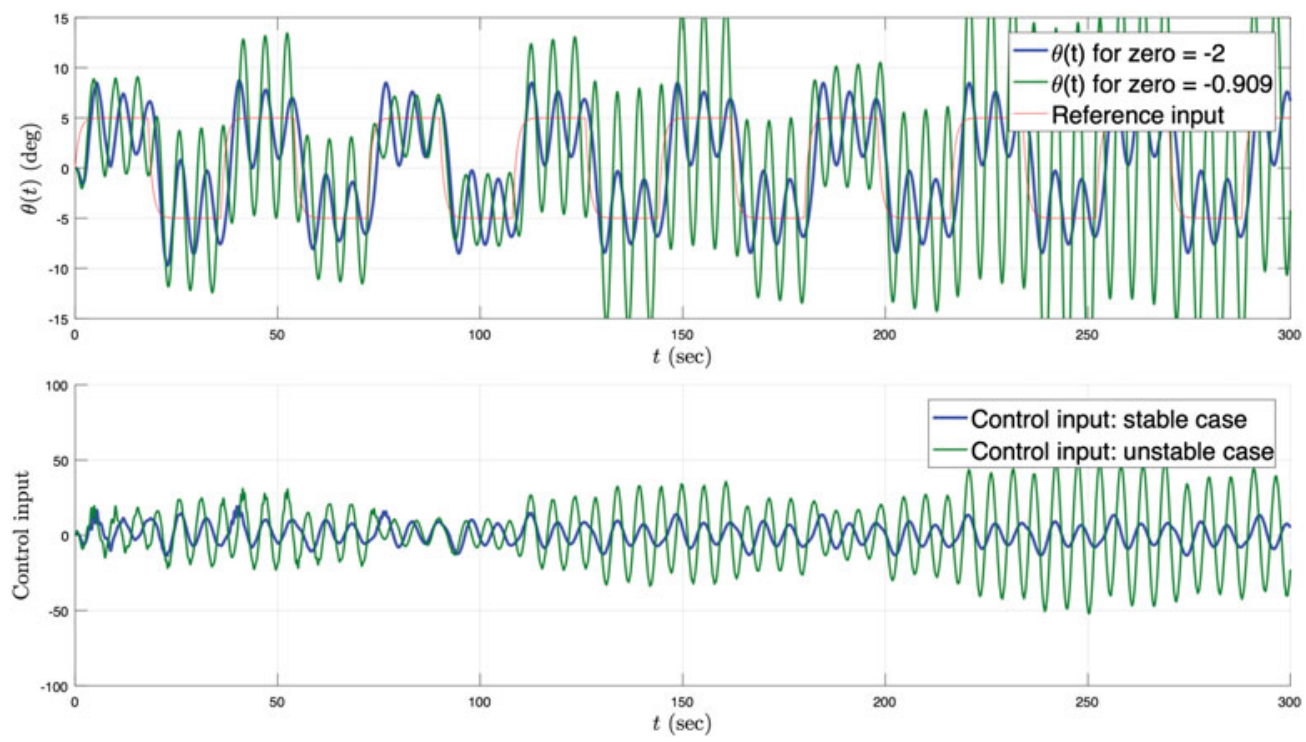

Figure 8. Tracking and control signal curves under uncertainties and MRAC for two different values of the pilot transfer function zero locations, $z=-2$ and $z=-0.909$, when $\mu=10$. 


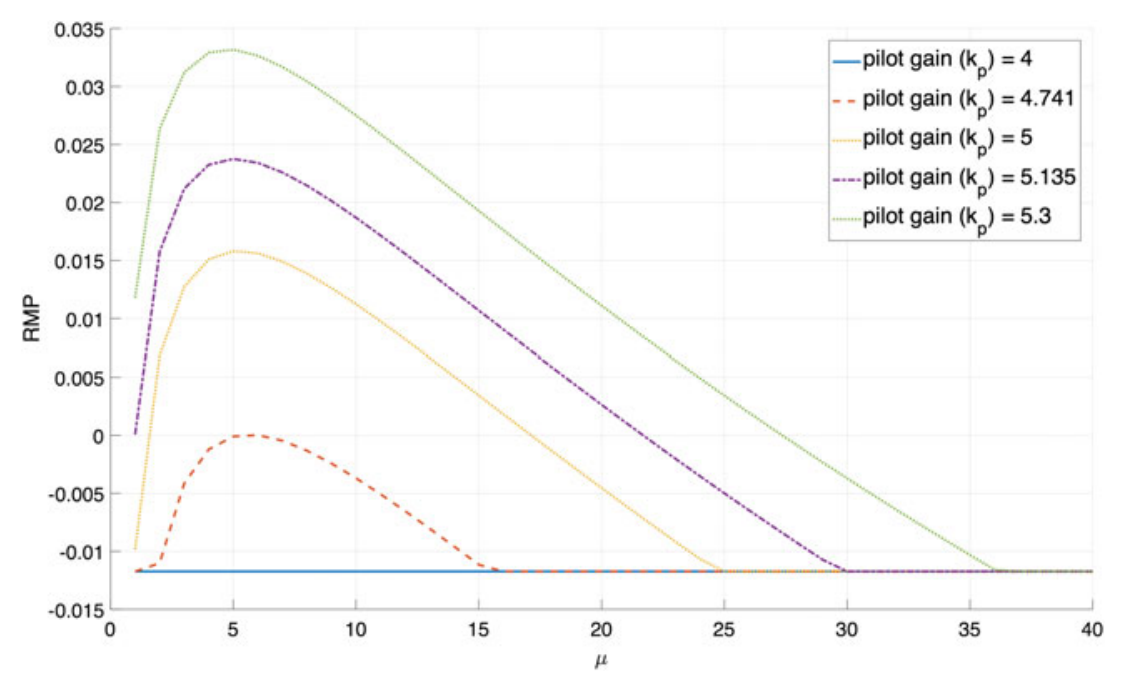

Figure 9. The location of the rightmost pole of (45) with respect to the control penalty variable $\mu$, for different pilot transfer function gain values.

Stability properties of the pilot-in-the-loop system depending on the nominal control penalty $\mu$ and the pilot gain $k_{p}$ is presented in Figure 9, where the RMP vs. $\mu$ is plotted for certain values of $k_{p}$. In these analyses, the pole and zero locations and time delay of the pilot transfer function are kept at their nominal values of $-0.2,-1$ and 0.5 , respectively. From the figure, stable-unstable-stable stability transition is once again observed for increasing values of $\mu$. On the other hand, it is seen that, similar to the trend for the pilot pole location, as the pilot gain increases, the $\mu$ stability region shrinks. As an example, it is predicted in Figure 9 that the closed-loop system will be stable for $k_{p}=4$ and unstable for $k_{p}=5$, when $\mu=10$. This is confirmed by the results presented in Figure 10, where time-domain tracking and control signal curves under uncertainties and MRAC are plotted for these gain values. While the settings in our simulations are not selected to address a specific application problem, predicted instability in the simulations due to human reaction delays and human high gain provides an interesting perspective and alignment with the well-known adverse effects of high gain of pilots on system stability, such as pilot-induced oscillations (Acosta et al., 2014; Yildiz \& Kolmanovsky, 2011; McRuer, 1992).
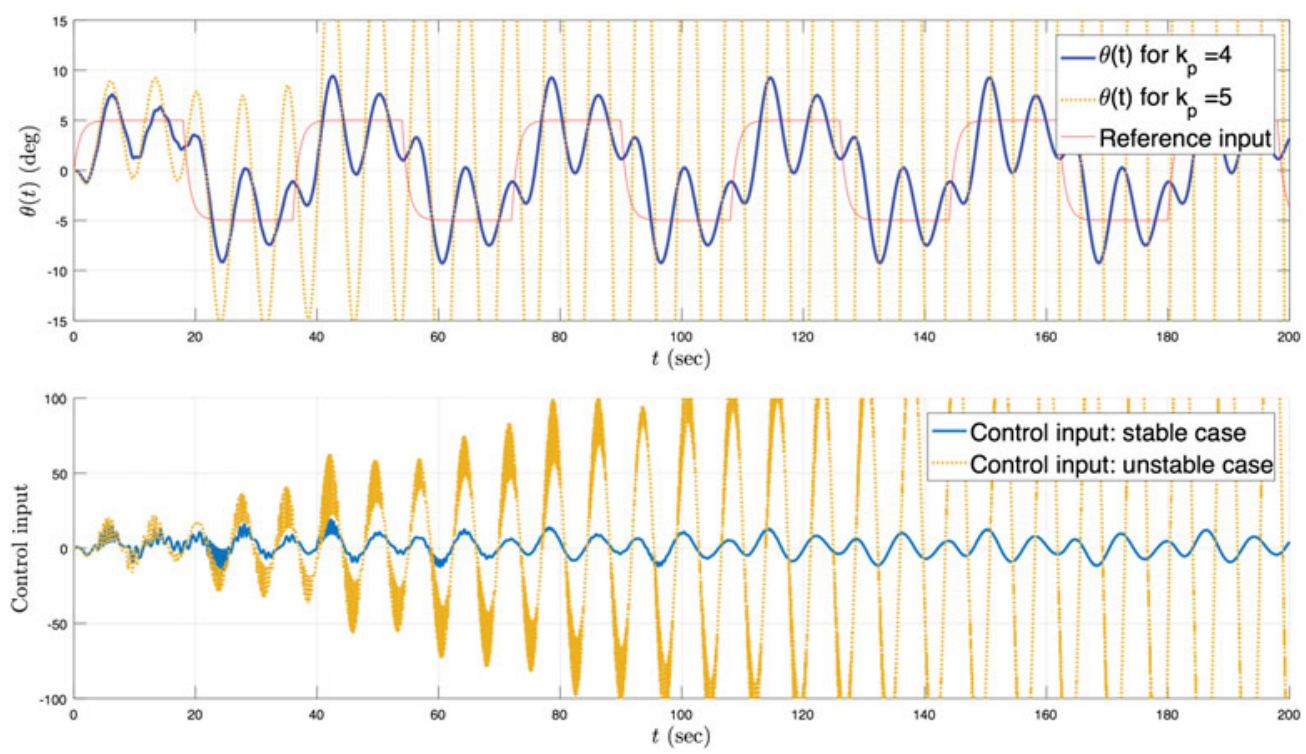

Figure 10. Tracking and control signal curves under uncertainties and MRAC for two different values of the pilot transfer function gain values, $k_{p}=4$ and $k_{p}=5$, when $\mu=10$. 


\section{Conclusion}

We analyse human-in-the-loop model reference adaptive control architectures and explicitly derive FSLs for both delay-independent and delay-dependent stability cases. Specifically, this stability limit results from the coupling between outer and inner-loop architectures, where the outer-loop portion includes the human dynamics modelled as a linear dynamical system with time delay and the inner-loop portion includes the uncertain dynamical system, the reference model, the parameter adjustment mechanism and the controller. In particular, a decoupling of the inner and outer loops enables one to prove the stability of each loop independently, which is then tailored together to declare the overall stability of the closed-loop system where the two loops are coupled. With this philosophy, not only the complex inner-outerloop dynamics is guaranteed to be stable, but it becomes possible to propose in a systematic way the tools needed to analyse and design the inner-outer-loop dynamics. The arising analysis points to several FSLs, which can be shown to be independent of the inner-loop adaptive controller but only depend on the human dynamics and a nominally designed reference model, enabling a clear view of what the influencing factors on such limits are. With this in mind, a number of simulation case studies are presented involving different designs for the reference model, human reaction delays, and against different levels of uncertainties, all demonstrating supportive results of the theoretical stability predictions. While the main focus of this study was to reveal stability limit of human-in-the-loop model reference adaptive control architectures, it is key to emphasise the modularity of the developed framework and how it decomposes the stability problem into manageable pieces. This offers a new perspective to handling control problems of nonlinear systems and whereby humans can be in the loop. Last but not least, several future studies are under consideration. Specifically, it is important to fine-tune the design parameters to render more desirable transient characteristics, to understand the interplay between the bandwidths of model reference control and human dynamics, and their impacts on the closed-loop system, and ultimately generalise the approach across a number of different nonlinear controllers, including sliding mode control and input-output linearisation.

\section{Note}

1. Although we consider a specific yet widely studied parameter adjustment mechanism given by (16), one can also consider other types of parameter adjustment mechanisms without changing the essence of this paper; for example, see Narendra and Annaswamy (1987), Ioannou and Kokotovic (1984), Pomet and Praly (1992), Yucelen and Calise (2010), Nguyen, Krishnakumar, and Boskovic (2008), Nguyen, Bakhtiari-Nejad, and Ishihira (2010), Yucelen, Calise, and Nguyen (2011), Calise and Yucelen (2012), Chowdhary and Johnson (2010), Chowdhary, Yucelen, Mühlegg, and Johnson (2013), Yucelen and Calise (2011), Yucelen and Haddad (2013), Yucelen, Gruenwald, and Muse (2015), Gruenwald and Yucelen (2015).

\section{Disclosure statement}

No potential conflict of interest was reported by the authors

\section{Funding}

This research was supported in part by the National Aeronautics and Space Administration under Grant NNX15AM51A.

\section{ORCID}

Tansel Yucelen (D http://orcid.org/0000-0003-1156-1877

\section{References}

Acosta, D. M., Yildiz, Y., Craun, R. W., Beard, S. D., Leonard, M. W., Hardy, G. H., \& Weinstein, M. (2014). Piloted evaluation of a control allocation technique to recover from pilotinduced oscillations. Journal of Aircraft, 52, 130-140.

Åström, K. J., \& Wittenmark, B. (2013). Adaptive control. Mineola, NY: Courier Corporation.

Bando, M., Hasebe, K., Nakanishi, K., \& Nakayama, A. (1998). Analysis of optimal velocity model with explicit delay. Physical Review E, 58, 5429-5435.

Bekiaris-Liberis, N., \& Krstic, M. (2010). Delay-adaptive feedback for linear feedforward systems. Systems and Control Letters, 59, 277-283.

Bellman, R., \& Cooke, K. (1963). Differential-difference equations. New York, NY: Academic Press.

Bernstein, D. S. (2009). Matrix mathematics: Theory, facts, and formulas (2nd ed.). Princeton, NJ: Princeton University Press.

Breda, D., Maset, S., \& Vermiglio, R. (2006). Pseudospectral differencing methods for characteristic roots of delay differential equations. SIAM Journal of Scientific Computing, 27, 482-495.

Breda, D. (2008). TRACE DDE. Retrieved from https:// users.dimi.uniud.it/dimitri.breda/research/software/.

Bresch-Pietri, D., \& Krstic, M. (2009). Adaptive trajectory tracking despite unknown input delay and plant parameters. Automatica, 45, 2074-2081.

Bryson, A. E. (1994). Control of spacecraft and aircraft. Princeton, NJ: Princeton University Press.

Calise, A. J., \& Yucelen, T. (2012). Adaptive loop transfer recovery. Journal of Guidance, Control, and Dynamics, 35, 807815.

Chen, J., \& Latchman, H. A. (1995). Frequency sweeping tests for stability independent of delay. IEEE Transactions on Automatic Control, 40, 1640-1645. 
Chowdhary, G., \& Johnson, E. N. (2010). Theory and flight test validation of a concurrent learning adaptive controller. AIAA Journal of Guidance, Control, and Dynamics, 34, 592607.

Chowdhary, G., Yucelen, T., Mühlegg, M., \& Johnson, E. N. (2013). Concurrent learning adaptive control of linear systems with exponentially convergent bounds. International Journal of Adaptive Control and Signal Processing, 27, 280301.

Engelborghs, K., Luzyanina, T., \& Roose, D. (2000). Numerical bifurcation analysis of delay differential equations using DDE-BIFTOOL. ACM Transactions on Mathematical Software, $1,1-21$.

Green, M. (2000). "How long does it take to stop?" Methodological analysis of driver perception-brake times. Transportation Human Factors, 2, 195-216.

Gruenwald, B., \& Yucelen, T. (2015). On transient performance improvement of adaptive control architectures. International Journal of Control, 88, 2305-2315.

Gu, K., Kharitonov, V. L., \& Chen, J. (2003). Stability of timedelay systems. Boston, MA: Birkhauser.

Haddad, W. M., Chellaboina, V., \& Kablar, N. A. (1999). Nonlinear impulsive dynamical systems. Part I: Stability and dissipativity. In Proceedings of the IEEE Conference on Decision and Control (pp. 4404-4422). Phoenix, AZ. [ Also in International Journal of Control (2001), 74, 1631-1658].

Helbing, D. (2001). Traffic and related self-driven many-particle systems. Reviews of Modern Physics, 73, 1067-1141.

Ioannou, P., \& Kokotovic, P. (1984). Instability analysis and improvement of robustness of adaptive control. Automatica, 20, 583-594.

Ioannou, P. A., \& Sun, J. (2012). Robust adaptive control. Mineola, NY: Courier Corporation.

Khalil, H. K. (1996). Nonlinear systems. Upper Saddle River, NJ: Prentice Hall.

Klyde, D. H., \& McRuer, D. (2009). Smart-cue and smart-gain concepts development to alleviate loss of control. Journal of Guidance, Control, and Dynamics, 32, 1409-1417.

Krstic, M. (1994). Delay compensation for nonlinear, adaptive, and PDE systems. Boston, MA: Birkhauser.

Lavretsky, E., \& Wise, K. (2012). Robust and adaptive control: With aerospace applications. London: Springer-Verlag.

Lewis, F. L., Liu, K., \& Yesildirek, A. (1995). Neural net robot controller with guaranteed tracking performance. IEEE Transactions on Neural Networks, 6, 703-715.

Lewis, F. L., Yesildirek, A., \& Liu, K. (1996). Multilayer neuralnet robot controller with guaranteed tracking performance. IEEE Transactions on Neural Networks, 7, 388-399.

McRuer, D. (1992). Human dynamics and pilot-induced oscillations (Minta Martin lecture). Cambridge, MA: Massachusetts Institute of Technology.

McRuer, D. T. (1974). Mathematical models of human pilot behavior.No. AGARD-AG-188. Advisory group for Aerospace research and development neuilly-sur-seine (France).

Miller, C. J. (2011). Nonlinear dynamic inversion baseline control law: Architecture and performance predictions. AIAA Guidance, Navigation, and Control Conference, Portland, OR.

Narendra, K. S., \& Annaswamy, A. M. (1987). A new adaptive law for robust adaptation without persistent excitation. IEEE Transactions on Automatic Control, 32, 134-145.
Narendra, K. S., \& Annaswamy, A. M. (2012). Stable adaptive systems. Minoela, NY: Courier Corporation.

Nguyen, N., Bakhtiari-Nejad, M., \& Ishihira, A. (2010). Robust adaptive optimal control with large adaptive gain. Proceedings of the American Control Conference, St. Louis, MO.

Nguyen, N., Krishnakumar, K., \& Boskovic, J. (2008). An optimal control modification to model-reference adaptive control for fast adaptation. Proceedings of AIAA Guidance, Navigation, and Control Conference, Honolulu, HI.

Nguyen, N., \& Summers, E. (2011). On time delay margin estimation for adaptive control and robust modification adaptive laws. In AIAA Guidance, navigation, and control conference.

Nia, P. M., \& Sipahi, R. (2014, Delay-independent stable setpoint tracking in a LTI networked control system with two uncertain delays; design and experiments. In Proceedings of the American Control Conference, Portland, OR.

Niculescu, S. I., \& Annaswamy, A. M. (2003). An adaptive smith-controller for time-delay systems with relative degree $n \leq 2$. Systems and Control Letters, 49, , 347-358.

Ortega, R., \& Lozano, R. (1988). Globally stable adaptive controller for systems with delay. International Journal of Control, 47, 17-23.

Osburn, P. V., Whitaker, H. P., \& Kezer, A. (1961). New developments in the design of adaptive control systems. Washington, DC: Institute of Aeronautical Sciences.

Pepe, P., \& Jiang, Z. P. (2005). A Lyapunov-Krasovskii methodology for ISS of time-delay systems. In Proceedings of IEEE European Conference of Decision and Control, Seville, Spain.

Pepe, P., \& Jiang, Z. P. (2006). A Lyapunov-Krasovskii methodology for iISS of time-delay systems. In Proceedings of IEEE Conference of Decision and Control, San Diego, CA.

Pomet, J. B., \& Praly, L. (1992). Adaptive nonlinear regulation: Estimation from Lyapunov equation. IEEE Transactions on Automatic Control, 37, 729-740.

Richard, J. P. (2003). Time-delay systems: an overview of some recent advances and open problems. Automatica, 39, 16671694.

Richards, N. D., Adams, R. J., Klyde, D. H., \& Cogan, B. (2015). Flight-test evaluation of an adaptive controller for flying qualities specification and protection. Journal of Guidance, Control, and Dynamics, 38, 2241-2256.

Ryu, S., \& Andrisani, D. (2003). Longitudinal flying qualities prediction for nonlinear aircraft. Journal of Guidance, Control, and Dynamics, 26, 474-482.

Schmidt, D., \& Bacon, B. (1983). An optimal control approach to pilot/vehicle analysis and the Neal-Smith criteria. Journal of Guidance, Control, and Dynamics, 6, 339-347.

Sipahi, R., Niculescu, S. I., Abdallah, C. T., Michiels, W., \& Gu, K. (2011). Stability and stabilization of systems with time delay, limitations and opportunities. IEEE Control Systems Magazine, 31, 38-65.

Skogestad, S., \& Postlethwaite, I. (2007). Multivariable feedback control: Analysis and design. New York, NY: Wiley.

Sontag, E. D. (1998). Comments on integral variants of ISS. Systems and Control Letters, 34, 93-100.

Stépán, G. (1989). Retarded dynamical systems: Stability and characteristic function. Longman Scientific \& Technical. New York, NY: John Wiley \& Sons.

Stépán, G. (2009). Delay effects in brain dynamics. Philosophical Transactions of The Royal Society A - Mathematical Physical \& Engineering Sciences, 367, 1059-1062. 
Thurling, A. J. (2000). Improving UAV handling qualities using time delay compensation ( Technical report, DTIC Document), No. AFIT/GAB/ENY/00M-01. AIR FORCE INST OF TECH, WRIGHT-PATTERSON AFB, OH.

Treiber, M., Kesting, A., \& Helbing, D. (2006). Delays, inaccuracies and anticipation in microscopic traffic models. Physica A, 360, 71-88.

Trujillo, A., \& Gregory, I. (2013). Adaptive controller adaptation time and available control authority effects on piloting. (NASA Technical Reports Server). 17th International Symposium on Aviation Psychology, Dayton, $\mathrm{OH}$.

Trujillo, A. C., Gregory, I. M., \& Hempley, L. E. (2015). Adaptive state predictor based human operator modeling on longitudinal and lateral control. AIAA Modeling and Simulation Technologies Conference, Kissimmee, FL.

Verriest, E. I., \& Ivanov, A. F. (1994). Robust stability of systems with delayed feedback. Circuits, Systems and Signal Processing, 13, 213-222.

Vyhlidal, T., \& Zitek, P. (2009). Mapping based algorithm for large-scale computation of quasi-polynomial zeros. IEEE Transactions on Automatic Control, 54, 171-177.

Weinmann, A. (2012). Uncertain models and robust control. Wien, NY: Springer Science \& Business Media.

Whitaker, H. P., Yamron, J., \& Kezer, A. (1958). Design of model reference control systems for aircraft. Cambridge: Instrumentation Laboratory, Massachusetts Institute of Technology.

Witte, J. B. (2004). An investigation relating longitudinal pilotinduced oscillation tendency rating to describing function predictions for rate-limited actuators (Technical report, DTIC Document). No. AFIT/GAE/ENY/04-M16. AIR FORCE INST OF TECH WRIGHT-PATTERSON AFB OH SCHOOL OF ENGINEERING AND MANAGEMENT.

Yi, S., Nelson, P. W., \& Ulsoy, A. G. (2010). Time-delay systems: Analysis and control using the Lambert $W$ function. Singapore: World Scientific Publishing Company.

Yildiz, Y., Annaswamy, A., Kolmanovsky, I. V., \& Yanakiev, D. (2010). Adaptive posicast controller for time-delay systems with relative degree $n \leq 2$. Automatica, 46, 279-289.

Yildiz, Y., \& Kolmanovsky, I. (2011). Stability properties and cross-coupling performance of the control allocation scheme CAPIO. Journal of Guidance, Control, and Dynamics, 34, 1190-1196.

Yucelen, T., \& Calise, A. J. (2010). Kalman filter modification in adaptive control. Journal of Guidance, Control, and Dynamics, 33, 426-439.

Yucelen, T., \& Calise, A. J. (2011). Derivative-free model reference adaptive control. Journal of Guidance, Control, and Dynamics, 34, 933-950.

Yucelen, T., Calise, A. J., \& Nguyen, N. T. (2011). "Evaluation of derivative-free adaptive controller with optimal control modification. AIAA Guidance, Navigation, and Control Conference, Portland, OR.

Yucelen, T., De La Torre, G., \& Johnson, E. N. (2014). Improving transient performance of adaptive control architectures using frequency-limited system error dynamics. International Journal of Control, 87, 2383-2397.

Yucelen, T., Gruenwald, B., \& Muse, J. A. (2015). A direct uncertainty minimization framework in model reference adaptive control. AIAA Guidance, Navigation, and Control Conference, Kissimmee, FL.
Yucelen, T., \& Haddad, W. M. (2012). A robust adaptive control architecture for disturbance rejection and uncertainty suppression with $\mathcal{L}_{\infty}$ transient and steady-state performance guarantees. International Journal of Adaptive Control and Signal Processing, 26, 1024-1055.

Yucelen, T., \& Haddad, W. M. (2013). Low-frequency learning and fast adaptation in model reference adaptive control. IEEE Transactions on Automatic Control, 58, 1080-1085.

Zhou, K., \& Doyle, J. C. (1998). Essentials of robust control. Upper Saddle River, NJ: Prentice hall.

Zhou, K., Doyle, J. C., \& Glover, K. (1996). Robust and optimal control. Upper Saddle River, NJ: Prentice Hall.

\section{Appendix 1}

Let $a$ and $b$ be two $n$-dimensional column vectors with real entries. Then the matrix $M=b a^{\mathrm{T}}$ is rank one, and hence, it has $n-1$ number of zero eigenvalues, $\lambda_{\ell}(M)=0$, $\ell=1, \ldots, n-1$ and a non-zero eigenvalue $\lambda_{n}(M) \neq 0$. In this case, the well-known property $\operatorname{tr}(M)=\sum_{\ell=1}^{n} \lambda_{\ell}(M)$ reduces to $\operatorname{tr}(M)=\lambda_{n}(M)$. Moreover, it is easy to see that $\operatorname{tr}(M)=a^{\mathrm{T}} b$. Therefore, the spectral radius of $M$, which is given by $\rho\left(b a^{\mathrm{T}}\right)=\left|\lambda_{n}(M)\right|$, is equal to $\left|a^{\mathrm{T}} b\right|$.

\section{Appendix 2}

We start with the following lemma from Bernstein (2009).

Lemma B.1: Let $A_{1} \in \mathbb{R}^{n \times n}, A_{2} \in \mathbb{R}^{n \times m}, A_{3} \in \mathbb{R}^{m \times n}$ and $A_{4} \in \mathbb{R}^{m \times m}$. If $A_{1}$ and $A_{4}-A_{3} A_{1}{ }^{-1} A_{2}$ are non-singular, then

$$
\left[\begin{array}{ll}
A_{1} & A_{2} \\
A_{3} & A_{4}
\end{array}\right]^{-1}=\left[\begin{array}{ll}
M_{1} & M_{2} \\
M_{3} & M_{4}
\end{array}\right]
$$

where $M_{2}=-A_{1}^{-1} A_{2}\left(A_{4}-A_{3} A_{1}^{-1} A_{2}\right)^{-1}$.

Next, let $K=\left[K_{1}, K_{2}\right]$, where $K_{1} \in \mathbb{R}^{m \times n_{\mathrm{p}}}$ and $K_{2} \in$ $\mathbb{R}^{m \times n_{\mathrm{c}}}$, and assume that $A_{p}-B_{p} K_{1}$ and $E_{\mathrm{p}}\left(A_{\mathrm{p}}-\right.$ $\left.B_{\mathrm{p}} K_{1}\right)^{-1} B_{\mathrm{p}} K_{2}$ are nonsingular matrices. Note that this is without loss of much generality since in general these matrices are indeed nonsingular especially when $m \leq n_{p}$.

Lemma B.2: Given $A_{r}, B_{r}$, and $E_{h}$ defined in Section 2 with $E_{\mathrm{h}_{\mathrm{p}}}=E_{\mathrm{p}}, E_{\mathrm{h}} A_{\mathrm{r}}^{-1} B_{\mathrm{r}}=-I$ holds.

Proof: It follows from $A_{\mathrm{r}}=A-B K$ that

$$
A_{\mathrm{r}}=\left[\begin{array}{cc}
A_{\mathrm{p}} & 0 \\
E_{\mathrm{p}} & 0
\end{array}\right]-\left[\begin{array}{c}
B_{\mathrm{p}} \\
0
\end{array}\right]\left[K_{1}, K_{2}\right]=\left[\begin{array}{cc}
X & Y \\
E_{\mathrm{p}} & 0
\end{array}\right],
$$

where $X \triangleq A_{\mathrm{p}}-B_{\mathrm{p}} K_{1}$ and $Y \triangleq B_{\mathrm{p}} K_{2}$. Letting

$$
A_{\mathrm{r}}^{-1} \triangleq\left[\begin{array}{ll}
M_{1} & M_{2} \\
M_{3} & M_{4}
\end{array}\right]
$$


it follows from Lemma B.1 that $M_{2}=X^{-1} Y\left(E_{\mathrm{p}} X^{-1} Y\right)^{-1}$. Using $B_{\mathrm{r}}$ and $E_{\mathrm{h}}$, one can further write

$$
\begin{aligned}
E_{\mathrm{h}} A_{\mathrm{r}}^{-1} B_{\mathrm{r}} & =\left[\begin{array}{ll}
E_{\mathrm{h}_{\mathrm{p}}} & 0
\end{array}\right]\left[\begin{array}{ll}
M_{1} & M_{2} \\
M_{3} & M_{4}
\end{array}\right]\left[\begin{array}{c}
0 \\
-I
\end{array}\right] \\
& =\left[\begin{array}{ll}
E_{\mathrm{p}} & 0
\end{array}\right]\left[\begin{array}{l}
-M_{2} \\
-M_{4}
\end{array}\right]=-E_{\mathrm{p}} M_{2},
\end{aligned}
$$

when $E_{\mathrm{h}_{\mathrm{p}}}=E_{\mathrm{p}}$. Finally, it is now immediate that

$$
E_{\mathrm{h}} A_{\mathrm{r}}^{-1} B_{\mathrm{r}}=-E_{\mathrm{p}} M_{2}=-E_{\mathrm{p}} X^{-1} Y\left(E_{\mathrm{p}} X^{-1} Y\right)^{-1}=-I,
$$

which completes the proof.
Notice for the SISO case considered in Corollary 3.2 that $E_{\mathrm{h}} A_{\mathrm{r}}^{-1} B_{\mathrm{r}}=-1$. 